\title{
The Physical States of Fermi Systems
}

\author{
SAlVADOR MiraCle-SOLE \\ C.N.R.S., Marseille \\ DEREK W. ROBINSON \\ Faculté des Sciences, Luminy, Marseille \\ Received June 11, 1969
}

\begin{abstract}
A classification of all translationally invariant states over the algebra of anticommutation relations which satisfy criteria of finite mean density, finite mean kinetic energy, and finite mean entropy is given. It is demonstrated that these concepts can be discussed in terms of affine, semi-continuous, functionals which respect the barycentric decompositions of invariant states. Many other pertinent results, both local and global, are derived.
\end{abstract}

\section{Introduction}

It is generally accepted that the states of physical systems can be theoretically identified with the mathematical states over suitably chosen $C^{*}$-algebras, the algebras of observables. For reasons of economy, however, one usually restricts attention to the subset of states which satisfy general conditions characteristic of the particular physical setting under consideration. In particular in statistical mechanics it is to be expected that a broad enough description is provided by states satisfying requirements of spatial homogeneity together with certain density restrictions. Following the original papers [1-3], the homogeneity requirements have been extensively studied to give a classification of invariant, periodic, and almost-periodic states. The density restrictions, which were particularly emphasized by Ruelle [3], have however attracted less attention. It is the purpose of the present paper to attempt to fill this gap by giving a detailed discussion of these restrictions in the case of Fermi systems. We consider the particle, kinetic energy, and entropy densities.

Each state $\varrho$ over a $C^{*}$-algebra $\mathfrak{U}$ determines a representation $\pi_{\varrho}$ of $\mathfrak{U}$ on a Hilbert space $\mathscr{H}_{\varrho}$ and a cyclic vector $\Omega_{\varrho}$ such that

$$
\varrho(A)=\left(\Omega_{\varrho}, \pi_{\varrho}(A) \Omega_{\varrho}\right) \text {. }
$$

If $\mathfrak{A}$ is the algebra associated with the canonical anti-commutation relations then the concept of particle density is usually discussed in terms 
of positive, but unbounded, number operators $N_{A, e}$ on $\mathscr{H}_{e}$ which are defined for each finite subsystem $A$. Of course such operators exist only for certain states. We will principally discuss a situation which is a little more restrictive. We consider states such that the $N_{A, e}$ exist with the property that

$$
N_{\Lambda}(\varrho)=\left(\Omega_{\varrho}, N_{\Lambda, \varrho} \Omega_{\varrho}\right)<+\infty
$$

i.e. $\Omega_{\varrho}$ must be in the domain of the $\left(N_{A, \varrho}\right)^{\frac{1}{2}}$. States of this nature are physically interpretable as states of finite particle density. The discussion of these states is simplified by extending the functionals $N_{A}$ defined above by setting $N_{\Lambda}(\varrho)=+\infty$ if $\varrho$ is not a state of finite particle density. It is shown that the $N_{A}$ are affine lower semi-continuous functionals and that the properties of the states of finite mean density are completely determined by the properties of these functionals. Further if $\varrho$ is translationally invariant one finds that $N_{A}(\varrho)$ is proportional to the volume $V(\Lambda)$ of the subsystem $\Lambda$ and the proportionality functional determines the properties of homogeneous states of finite density. This study can finally be reframed in terms of density operators $N_{e}$ which we show to be proportional to the identity operator on $\mathscr{H}_{\varrho}$ in the case that $\varrho$ is an ergodic invariant state of finite density.

Discussion of the states of finite kinetic energy density follows essentially the same lines as the above. A number of new difficulties arise which are mostly traceable to the lack of uniqueness of boundary conditions in the description of finite systems of freely moving particles. Note that our investigation of this latter problem is not motivated by interest in the free system but by the hope that the states considered should be useful both in the discussion of the time development of interacting Fermi systems and in the description of equilibrium phenomena. In particular we proceed to demonstrate that a satisfactory assignment of mean entropy can be given for the states of finite particle and kinetic energy densities.

\section{The Algebra of Observables}

We begin by giving a brief survey of the properties of the $C^{*}$-algebra used to describe systems of fermions i.e. the $C^{*}$-algebra associated with the canonical anti-commutation relations. We specialize to the framework encountered in statistical mechanics by taking the Euclidean space in $v$-dimensions $R^{v}$ as underlying configuration space and recall the properties most relevant to this setting and the subsequent analysis.

The $C^{*}$-algebra $\mathfrak{A}$ associated with the canonical anti-commutation relations is algebraically generated by elements $a(f)$ and their adjoints 
$a(g)^{*}$ which are defined for all $f, g \in L^{2}\left(R^{v}\right)$, the Hilbert space of complex square integrable functions over $R^{v}$. The elements $a(f)$ and $a(g)^{*}$ are assumed to depend linearly and anti linearly on $f$ and $g$ respectively and also satisfy the algebraic relations

$$
\begin{gathered}
{\left[a(f), a(g)^{*}\right]_{+}=a(f) a(g)^{*}+a(g)^{*} a(f)=(g, f),} \\
{[a(f), a(g)]_{+}=a(f) a(g)+a(g) a(f)=0,}
\end{gathered}
$$

where (,) denotes the scalar product in $L^{2}\left(R^{v}\right)$. The algebraic norm \|\| of the element $a(f)$ coincides with the $L^{2}$-norm of the function $f$ i.e.

$$
\|a(f)\|=|f|_{2}=\left\|a(f)^{*}\right\| \text {. }
$$

The algebra $\mathfrak{Q}$ is both separable and simple (for a detailed discussion of the construction of $\mathfrak{Q}$ and its properties the reader is referred to [4]).

If $\Lambda$ is a bounded open set in $R^{v}$ then the elements $a\left(f_{A}\right), a\left(g_{A}\right)^{*}$, which are defined for all $f_{\Lambda}, g_{\Lambda} \in L^{2}(\Lambda)$ generate a $C^{*}$-subalgebra $\mathfrak{U}(\Lambda)$ of $\mathfrak{A}$. The family of subalgebras defined in this manner will be referred to as local algebras. They satisfy the isotony relationship $\mathfrak{A}(\Lambda) \subset \mathfrak{U}\left(\Lambda^{\prime}\right)$ if $\Lambda \subset \Lambda^{\prime}$.

Two groups of automorphisms of $\mathfrak{I}$ are of special importance. The first is the group $R^{v}$ of space translations. There exists a representation $\tau$ of the group $R^{v}$ as strongly continuous automorphisms of $\mathfrak{A}$. The action of this group on the generating elements of $\mathfrak{A}$ is defined by

$$
\tau_{x}\left(a\left(f_{A}\right)\right)=a\left(f_{A+x}\right), \quad x \in R^{v}
$$

where $f_{\Lambda+x} \in L^{2}(\Lambda+x)$ is given by

$$
f_{A+x}(y)=f_{A}(y-x) \text {. }
$$

Secondly there exists a strongly continuous representation $\sigma$ of the compact one-parameter group of gauge transformations of $\mathfrak{A}$. The action of this latter group on the generating elements of $\mathfrak{A}$ is defined by

$$
\sigma_{\alpha}(a(f))=a\left(f_{\alpha}\right), \quad 0 \leqq \alpha \leqq 2 \pi
$$

where $f_{\alpha}$ is given by

$$
f_{\alpha}(x)=e^{-i \alpha} f(x) .
$$

The gauge group can be used to classify odd and even elements of $\mathfrak{A}$ as follows. An element $A \in \mathfrak{U}$ is defined to be odd, or even, if the relation

$$
\sigma_{\pi}(A)=\mp A
$$

holds with the minus, or plus, sign, respectively. A unique decomposition of a general element $A \in \mathfrak{U}$ as a sum of odd and even elements is given by

$$
A=\frac{1}{2}\left(A-\sigma_{\pi}(A)\right)+\frac{1}{2}\left(A+\sigma_{\pi}(A)\right) .
$$


The even elements of $\mathfrak{U}$ form a $C^{*}$-subalgebra $\mathfrak{U}_{E} \subset \mathfrak{U}$ whilst the odd elements span a Banach subspace of $\mathfrak{U}$. This classification can of course be made for the local subalgebras $\mathfrak{A}(\Lambda)$ and is useful for a general presentation of the commutation properties of elements of $\mathfrak{U}$. These properties are included in the following list which also summarizes the features discussed above.

The fermi algebra $\mathfrak{A}$ and its local subalgebras $\mathfrak{A}(\Lambda), \Lambda \subset R^{\nu}$, possess the following structural properties.

0. $\mathfrak{A}=\overline{\left\{\mathfrak{A}(\Lambda), \Lambda \subset R^{v}\right\}}$ is separable and simple.

1. $\mathfrak{U}\left(\Lambda_{1}\right) \subset \mathfrak{U}\left(\Lambda_{2}\right)$ if $\Lambda_{1} \subset \Lambda_{2}$.

2. If $\Lambda_{1} \cap \Lambda_{2}=\theta$ and $A_{1} \in \mathfrak{U}\left(\Lambda_{1}\right), A_{2} \in \mathfrak{U}\left(\Lambda_{2}\right)$ are both odd then $\left[A_{1}, A_{2}\right]_{+}=0$ if however $A_{1}$, or $A_{2}$, (or both) is even then

$$
\left[A_{1}, A_{2}\right]_{-}=A_{1} A_{2}-A_{2} A_{1}=0 \text {. }
$$

3. The group $R^{v}$ acts as a group of strongly continuous automorphisms of $\mathfrak{A}$ such that

$$
A \in \mathfrak{A}(\Lambda) \rightarrow \tau_{x} A \in \mathfrak{A}(\Lambda+x), \quad x \in R^{v} .
$$

4. If $A, B \in \mathfrak{A}$ are both odd then

$$
\left\|\left[A, \tau_{x} B\right]_{+}\right\| \underset{|x|=\infty}{\longrightarrow} 0
$$

if however either $A$, or $B$ is even then

$$
\left\|\left[A, \tau_{x} B\right]_{-}\right\| \underset{|x|=\infty}{\longrightarrow} 0 .
$$

In the statistical mechanics of fermi systems the elements of $\mathfrak{A}$ are considered to describe the physical observables and the possible states of the system are identified with the mathematical states over $\mathfrak{A}$ or subclasses of these states. We next consider the details of this description.

\section{Physical States}

The states, the normalized positive linear functionals, $\varrho$ over $\mathfrak{A}$ form a convex weakly compact subset $E$ of the dual $\mathfrak{A}^{\prime}$ of $\mathfrak{A}$. Associated with each state $\varrho$ by the Gelfand-Segal construction is a representation $\pi_{\varrho}$ of $\mathfrak{U}$ as bounded operators acting on a Hilbert space $\mathscr{H}_{Q}$ with cyclic vector $\Omega_{\varrho}$ such that

$$
\varrho(A)=\left(\Omega_{\varrho}, \pi_{\varrho}(A) \Omega_{\varrho}\right) .
$$

As $\mathfrak{A}$ is algebraically generated by the local algebras $\mathfrak{A}(\Lambda)$ each state $\varrho$ is determined by its restriction to $\left\{\mathfrak{Q}(\Lambda) ; \Lambda \subset R^{v}\right\}$. We begin our discussion of the physical restrictions to be imposed on the states $\varrho$ by considering the basic irreducible Fock representations of the local algebras $\mathfrak{A}(\Lambda)$ and their associated vector states. 
Let $L_{-}^{2}\left(\Lambda^{n}\right)$ denote the Hilbert space of totally antisymmetric square integrable functions of $n$ points in $\Lambda$ and define the Fock space $\mathscr{H}_{F}(\Lambda)$ by

$$
\mathscr{H}_{F}(\Lambda)=\bigoplus_{n \geqq 0} L_{-}^{2}\left(\Lambda^{n}\right),
$$

i.e. an element of $\mathscr{H}_{F}(\Lambda)$ is a sequence $\Psi=\left(\Psi^{(n)}\right)_{n \geqq 0}$ where $\Psi^{(0)}$ is a complex scalar, $\Psi^{(n)} \in L_{-}^{2}\left(\Lambda^{n}\right)$ for $n \geqq 1$, and such that

$$
(\Psi, \Psi)=\left|\Psi^{(0)}\right|^{2}+\sum \int d x_{1} \ldots d x_{n}\left|\Psi^{(n)}\left(x_{1}, \ldots x_{n}\right)\right|^{2}<+\infty .
$$

The Fock representation $\pi_{\Lambda}$ of $\mathfrak{U}(\Lambda)$ is defined on $\mathscr{H}_{F}(\Lambda)$ by prescribing the action of the generating elements as follows:

$$
\begin{aligned}
\left(\pi_{A}(a(f)) \Psi\right)^{(n)}\left(x_{1}, \ldots x_{n}\right) & =(n+1)^{\frac{1}{2}} \int d x f(x) \Psi^{(n)}\left(x_{1} \ldots x_{n}\right), \\
\left(\pi_{\Lambda}\left(a(f)^{*}\right) \Psi\right)^{(n)}\left(x_{1}, \ldots x_{n}\right) & =n^{-\frac{1}{2}} \sum_{m=1}^{n}(-1)^{m-1} f\left(x_{m}\right) \Psi^{(n-1)}\left(x_{1}, \ldots \hat{x}_{m} \ldots x_{n}\right)
\end{aligned}
$$

where $\hat{x}_{m}$ denotes that the argument $x_{m}$ is omitted. Physically $\pi_{\Lambda}(a(f))$ and $\pi_{\Lambda}\left(a(g)^{*}\right)$ are interpreted as operators which annihilate and create particles and a vector $\Psi^{(n)}$ represents a state of $n$ particles. This last point may be made more explicit by the introduction of a positive self adjoint operator $N_{\Lambda}$ on $\mathscr{H}_{F}(\Lambda)$ which measures particle number by the definition

$$
\left(N_{\Lambda} \Psi\right)^{(n)}\left(x_{1} \ldots x_{n}\right)=n \Psi^{(n)}\left(x_{1}, \ldots x_{n}\right) .
$$

Alternatively $N_{A}$ can be characterised by the following criterion. Let $\mathscr{F}=\left\{f_{\Lambda}^{i}\right\}_{i \geqq 1}$ be a complete orthonormal basis of functions $f_{\Lambda}^{i} \in L^{2}(\Lambda)$ and define $\mathscr{D}_{\mathscr{F}}$ by

$$
\mathscr{D}_{\mathscr{F}}=\left\{\Psi ; \Psi \in \mathscr{H}_{F}(\Lambda), \sum_{i=1}^{\infty}\left(\Psi, \pi_{\Lambda}\left(a\left(f_{\Lambda}^{i}\right)^{*} a\left(f_{\Lambda}^{i}\right)\right) \Psi\right)<+\infty\right\} .
$$

Now from [5], we know that for $\Phi, \Psi \in \mathscr{D}_{\mathscr{F}}$

$$
\left(\Phi, N_{\Lambda} \Psi\right)=\sum_{i=1}^{\infty}\left(\Phi, \pi_{\Lambda}\left(a\left(f_{\Lambda}^{i}\right)^{*} a\left(f_{\Lambda}^{i}\right)\right) \Psi\right)
$$

and further that $\mathscr{D}_{\mathscr{F}}=\mathscr{D}\left(N_{\lambda}^{\frac{1}{2}}\right)$ the domain of $N_{\lambda}^{\frac{1}{2}}$, is independent of the basis. Finally concerning $N_{A}$ we note that if we introduce the group $U_{A}$ of unitary operators on $\mathscr{H}_{F}(\Lambda)$ by the definition

$$
U_{\Lambda}(\alpha)=\exp \left\{i N_{\Lambda} \alpha\right\}, \quad 0 \leqq \alpha \leqq 2 \pi
$$

then the group $U_{A}$ implement the gauge automorphisms

$$
U_{\Lambda}(\alpha) \pi_{\Lambda}(a(f)) U_{\Lambda}(\alpha)^{-1}=\pi_{\Lambda}\left(\sigma_{\alpha}(a(f))\right) \quad \text { etc. ... }
$$

Thus the Fock representation of $\mathfrak{A}(\Lambda)$ and its vector states describe a finite number of particles in the finite region $\Lambda$ of configuration space. 
In statistical mechanics one would like to restrict ones attention to just those states $\varrho$ over $\mathscr{Q}$ which have this property for all $\Lambda \subset R^{v}$. Fortunately this form of restriction can be well characterised mathematically and the relevant results are contained in the following proposition which is a summary of known results [6-9].

Proposition 1. Let $\varrho$ be a state over $\mathfrak{A}(\Lambda)$ and $\left(\pi_{e}, \mathscr{H}_{\varrho}, \Omega_{\varrho}\right)$ the representation, Hilhert space, and cyclic vector, associated with $\varrho$ by the GelfandSegal construction. The following conditions are equivalent.

1. The representation $\pi_{e}$ is a sum of copies of the Fock representation.

2. There exists a density matrix $\varrho_{A}$ on $\mathscr{H}_{F}(\Lambda)$ such that

$$
\varrho(A)=\operatorname{Tr}_{\mathscr{H}_{F}(A)}\left(\varrho_{A} \pi_{A}(A)\right), \quad A \in \mathfrak{A}(A) .
$$

3. There exists an orthonormal basis $\left\{f_{A}^{i}\right\}_{i \geqq 1}$ in $L^{2}(A)$ such that the linear variety $\mathscr{D}_{Q} \in \mathscr{H}_{0}$ defined by

is dense in $\mathscr{H}_{0}$.

$$
\mathscr{D}_{Q}=\left\{\Psi ; \Psi \in \mathscr{H}_{Q}, \sum_{i=1}^{\infty}\left(\Psi, \pi_{e}\left(a\left(f_{A}^{i}\right)^{*} a\left(f_{A}^{i}\right)\right) \Psi\right)<\infty\right\}
$$

4. There exists an orthonormal basis $\left\{f_{A}^{i}\right\}_{i \geqq 1}$ in $L^{2}(\Lambda)$ such that

$$
\sum_{i=1}^{\infty}\left(\Psi, \pi_{e}\left(a\left(f_{A}^{i}\right)^{*} a\left(f_{A}^{i}\right)\right) \Psi\right)<+\infty
$$

for one vector $\Psi \in \mathscr{H}_{\varrho}$ cyclic for $\pi_{\varrho}$.

5. There exists a one-parameter strongly continuous group $U_{\varrho}$ of unitary operators on $\mathscr{H}_{Q}$ which implement the gauge automorphisms i.e.

$$
U_{\underline{\varrho}}(\alpha) \pi_{\varrho}(A) U_{\varrho}(\alpha)^{-1}=\pi_{\underline{\varrho}}\left(\sigma_{\alpha} A\right), \quad A \in \mathfrak{A}(A), 0 \leqq \alpha \leqq 2 \pi
$$

and such that the infinitesimal generator $G$, defined by $U_{Q}(\alpha)=\exp \{i G \alpha\}$ is semi-bounded.

The equivalence of Conditions 1,2 and 3 is proved in $[6,7]$. These conditions obviously imply 4 but the converse statement is also true as is remarked in Footnote 8 of [7] without proof. As this implication is of importance to us in the sequel we give the following proof.

Assuming Condition 4 it is sufficient to deduce that

$$
N\left(\pi_{\varrho}(A) \Psi, \pi_{\varrho}(A) \Psi\right)=\sum_{i=1}^{\infty}\left(\pi_{\varrho}(A) \Psi, \pi_{\varrho}\left(a\left(f_{A}^{i}\right)^{*} a\left(f_{A}^{i}\right)\right) \pi_{\varrho}(A) \Psi_{\varrho}\right)<+\infty
$$

for $A \in \mathfrak{Q}(A)$ a polynomial in creation and annihilation operators. But noting that

$$
\begin{aligned}
N\left(\pi_{\varrho}\left(A_{1}+A_{2}\right) \Psi, \pi_{\varrho}\left(A_{1}+A_{2}\right) \Psi\right) \leqq & N\left(\pi_{\varrho}\left(A_{1}\right) \Psi, \pi_{\varrho}\left(A_{1}\right) \Psi\right)+ \\
& +2 N\left(\pi_{\varrho}\left(A_{2}\right) \Psi, \pi_{\varrho}\left(A_{2}\right) \Psi\right)
\end{aligned}
$$


it is sufficient to prove the statement for monomials. Now let $A$ be given by

$$
A=a\left(f_{A}^{i_{1}}\right)^{*} \ldots a\left(f_{A}^{i_{1}}\right)^{*} a\left(f_{A}^{j_{1}}\right) \ldots a\left(f_{A}^{j_{s}}\right)
$$

and note that

$$
a\left(f_{A}^{i}\right) A=\left[a\left(f_{A}^{i}\right), A\right]_{(-1)^{r-1}}-(-1)^{r s} A a\left(f_{A}^{i}\right)
$$

where the (anti-)commutator occurring on the right hand side vanishes unless $i=i_{p}$ for $1 \leqq p \leqq r$. Hence one immediately finds that

$$
\begin{aligned}
\sum_{i=1}^{\infty}\left(\pi_{e}(A)\right. & \left.\Psi, \pi_{e}\left(a\left(f_{A}^{i}\right)^{*} a\left(f_{A}^{i}\right)\right) \pi_{e}(A) \Psi\right) \\
& \leqq 4 r+\sum_{i=1}^{\infty}\left(\Psi, \pi_{e}\left(a\left(f_{A}^{i}\right)^{*} a\left(f_{\Lambda}^{i}\right)\right) \Psi\right)<+\infty
\end{aligned}
$$

and Conditions 3 follows.

The equivalence of Conditions 1 and 5 is proved in $[8,9]$ for the algebra associated with the canonical commutation relations. The proof is valid in the present context and can even be simplified greatly.

The above proposition gives us a number of methods of characterising the states which describe a finite number of particles in each bounded region but as the physically relevant objects are the states the most useful criterion appears to be furnished by Condition 2 . A state $\varrho$ over $\mathfrak{A}(\Lambda)$ which is determined by a density matrix on Fock space is called normal (with respect to the Fock representation) and thus a state $\varrho$ over $\mathfrak{A}$ is called locally normal if the restriction of $\varrho$ to each local subalgebra $\mathfrak{A}(A)$ is normal. Each state $\varrho$ is determined by its restriction to $\left\{\mathfrak{A}(\Lambda) ; \Lambda \subset R^{\prime \prime}\right\}$ and hence a locally normal state is determined by a set of density matrices $\left\{\varrho_{A} ; \Lambda \subset R^{v}\right\}$. The isotony conditions relating the local subalgebras $\mathfrak{a}(\Lambda)$ place the following consistency conditions on the density matrices

$$
\operatorname{Tr}_{\mathscr{H}_{F}\left(A_{1} \cup A_{2}\right)}\left(\varrho_{A_{1} \cup A_{2}} \pi_{A_{1} \cup A_{2}}(A)\right)=\operatorname{Tr}_{\mathscr{H}_{F}\left(A_{1}\right)}\left(\varrho_{A_{1}} \pi_{A_{1}}(A)\right)
$$

for $\Lambda_{1} \cap \Lambda_{2}=0$ and $A \in \mathfrak{A}\left(\Lambda_{1}\right)$.

We will return to the discussion of special subsets of locally normal states in the following sections but we next recall the physical restrictions imposed by invariance criteria and the relevant mathematical results which have been derived. The translationally invariant states $\varrho$ over $\mathfrak{A}$ play a special role in statistical mechanics. These $R^{v}$ invariant states, i.e. the states such that

$$
\varrho\left(\tau_{x} A\right)=\varrho(A)
$$

for all $A \in \mathfrak{Q r}$ and $x \in R^{v}$, form a convex weakly compact subset $E \cap L_{R^{v}}^{\perp}$ of the state space $E$. The Gelfand-Segal construction applied to $\varrho \in E \cap L_{R^{v}}^{\perp}$ yields a unitary representation $U_{\varrho}$ of $R^{v}$ which implements 
the group $\tau$ of automorphisms i.e.

$$
U_{\varrho}(x) \pi_{\varrho}(A) U_{\varrho}(x)^{-1}=\pi_{\varrho}\left(\tau_{x} A\right)
$$

and leaves the cyclic vector $\Omega_{\varrho}$ invariant i.e.

$$
U_{\varrho}(x) \Omega_{\varrho}=\Omega_{\varrho} .
$$

In $[10]$ it is demonstrated that all translationally invariant states are even i.e.

$$
\varrho(A)=0
$$

if $A \in \mathfrak{A}$ is odd and this property and the commutation relations listed above imply that $\mathfrak{A}$ is $R^{v}$-abelian in the sense of Lanford and Ruelle [11] i.e. if $E_{\varrho}$ is the projector on the subspace of $\mathscr{H}_{\varrho}$ spanned by the $U_{\varrho}$ invariant vectors then the algebra $\left\{E_{\varrho} \pi_{e} E_{\varrho}\right\}$ is abelian. This last property then yields the following result.

Proposition 2. If $\varrho \in E \cap L_{R^{v}}^{\perp}$ is a translationally invariant state over $\mathfrak{A}$ then there exists a unique probability measure $\mu_{\varrho}$ with barycentre $\varrho$ concentrated on the extremal invariant states $\mathscr{E}\left(E \cap L_{R^{v}}^{\perp}\right)$ of $\mathfrak{A}$ i.e. @ has a unique integral decomposition

$$
\varrho(A)=\int d \mu_{\varrho}\left(\varrho^{\prime}\right) \varrho^{\prime}(A)
$$

in terms of extremal invariant states $\varrho^{\prime}$.

Further if $\varrho$ is locally normal then $\mu_{Q}$ is concentrated on extremal invariant states which are also locally normal.

The existence and uniqueness of the barycentric decomposition is proved in $[2,3,11]$ and the fact that the property of local normality is respected by this decomposition is a consequence of a more general result obtained in [3] and Proposition 1. Results of this same general nature are also derived in the following sections.

\section{Density and Mean Density}

In this section we consider the assignment of a density and mean density to states over the algebra $\mathfrak{A}$. Although the concept of a state with finite density is closely related to the notion of a locally normal state, i.e. a state describing a finite number of particles per unit volume of configuration space, the first concept is a little more stringent. If $\varrho$ is a locally normal state there exists a family of semibounded self adjoint number operators $\left\{N_{A}, \Lambda \subset R^{v}\right\}$ acting in the representation space $\mathscr{H}_{Q}$ associated with $\varrho$. Physically one wishes to interpret the cyclic vector $\Omega_{\varrho} \in \mathscr{H}_{\varrho}$ associated with $\varrho$ as the vector representing equilibrium of the system and hence the local density of $\varrho$ should be proportional to

$$
N_{\Lambda}(\varrho)=\left(\Omega_{\varrho}, N_{\Lambda} \Omega_{\varrho}\right)=\left(N_{\bar{\lambda}}^{\frac{1}{2}} \Omega_{\varrho}, N_{\bar{\lambda}}^{\frac{1}{2}} \Omega_{\varrho}\right)
$$


However unless $\Omega_{\varrho} \in \mathscr{D}\left(N_{\lambda}^{\frac{1}{2}}\right)$ this latter expression is meaningless. Thus the states with finite local density are a subclass of the locally normal states satisfying certain domain requirements. We will now study this class of states avoiding, as far as possible, the consideration of unbounded operators in Hilbert space. Instead we consider certain affine functionals over the state space of $\mathfrak{A}$ which we introduce through the following definition. The definition is motivated by Condition 4 of Proposition 1 .

Definition 1. Let $\mathscr{F}=\left\{f_{A}\right\}$ be a finite family of orthonormal functions $f_{\Lambda} \in L^{2}(\Lambda)$. The number functional $N_{\Lambda}$ is defined as a function from the state space $E_{\mathfrak{Q}(\Lambda)}$ of $\mathfrak{A}(\Lambda)$ to the interval $[0,+\infty]$ by

$$
N_{\Lambda}(\varrho)=\sup _{\mathscr{F}} \sum_{f_{\Lambda} \in \mathscr{F}} \varrho\left(a\left(f_{\Lambda}\right)^{*} a\left(f_{\Lambda}\right)\right) .
$$

In terms of the number functional introduced in this manner we can state the following theorem which is to a large extent a restatement of information contained in Proposition 1.

Theorem 1. $N_{\Lambda}$ is an affine lower semi-continuous function over the convex weakly compact set $E_{\mathfrak{Q}(\Lambda)}$ of states over $\mathfrak{A}(\Lambda)$. The following conditions are equivalent

1. $N_{\Lambda}(\varrho)<+\infty$.

2. $\varrho$ is normal with respect to the Fock representation, i.e. $\varrho$ is determined by a density matrix $\varrho_{A}$ on $\mathscr{H}_{F}(\Lambda)$, and

$$
\operatorname{Tr}_{\mathscr{H}_{F}(\Lambda)}\left(\varrho_{A} N_{A}\right)<+\infty
$$

where $N_{A}$ is the self-adjoint number operator associated with the Fock representation.

3. There exists in $L^{2}(\Lambda)$ a complete orthonormal basis $\left\{f_{\Lambda}^{i}\right\}_{i \geqq 1}$ such that

$$
\lim _{N \rightarrow \infty} \sum_{i=1}^{N} \varrho\left(a\left(f_{\Lambda}^{i}\right)^{*} a\left(f_{\Lambda}^{i}\right)\right)<+\infty .
$$

If these latter conditions are satisfied then

$$
N_{\Lambda}(\varrho)=\operatorname{Tr}_{\mathscr{H}_{F}(\Lambda)}\left(\varrho_{\Lambda} N_{\Lambda}\right)=\operatorname{Lim}_{N \rightarrow \infty} \sum_{i=1}^{N} \varrho\left(a\left(f_{\Lambda}^{i}\right)^{*} a\left(f_{\Lambda}^{i}\right)\right) .
$$

Proof. $N_{A}$ is defined as the supremum of a family of continuous functions and hence is lower semi-continuous.

Condition 1 trivially implies Condition 3 . The equivalence of Conditions 2 and 3 follows from using the Gelfand-Segal construction, Proposition 1, and the characterization of semi-bounded self adjoint operators supplied by [5]. In order to prove that Condition 2 implies Condition 1 introduce a complete orthonormal basis $\left\{f_{\Lambda}^{i}\right\}_{i \geqq 1}$ in $L^{2}(\Lambda)$ 
and let $\left\{\Psi^{j}\right\}_{j \geqq 1}$ be the vector states, in $\mathscr{H}_{F}(\Lambda)$, associated with the density matrix $\varrho_{A}$. From our discussion of the Fock representation we have

$$
\begin{aligned}
+\infty & >\operatorname{Tr}_{\mathscr{H}_{F}(A)}\left(\varrho_{A} N_{A}\right) \\
& =\sum_{j \geqq 1} \sup _{N} \sum_{i=1}^{N}\left(\Psi^{j}, \pi_{A}\left(a\left(f_{A}^{i}\right)^{*} a\left(f_{A}^{i}\right)\right) \Psi^{j}\right) \\
& \geqq \sup _{N} \sum_{j \geqq 1} \sum_{i=1}^{N}\left(\Psi^{i}, \pi_{A}\left(a\left(f_{A}^{i}\right)^{*} a\left(f_{A}^{i}\right)\right) \Psi^{j}\right) \\
& =\sup _{N} \sum_{i=1}^{N} \varrho\left(a\left(f_{A}^{i}\right)^{*} a\left(f_{A}^{i}\right)\right) .
\end{aligned}
$$

The validity of this inequality for all basis immediately implies $N_{A}(\varrho)<+\infty$. But now if the conditions are valid we have

$$
N_{A}(\varrho) \leqq \operatorname{Tr}_{\mathscr{H}_{F}(A)}\left(\varrho_{A} N_{A}\right)=\sum_{i=1}^{\infty} \varrho\left(a\left(f_{A}^{i}\right)^{*} a\left(f_{A}^{i}\right)\right) \leqq N_{A}(\varrho)
$$

where the middle equality follows from Proposition 1 and [5].

Finally we see from Condition 3 that

$$
N_{A}\left(\lambda \varrho_{1}+(1-\lambda) \varrho_{2}\right)=\lambda N_{A}\left(\varrho_{1}\right)+(1-\lambda) N_{A}\left(\varrho_{2}\right), \quad 0<\lambda<1
$$

if $N_{A}\left(\varrho_{1}\right)<+\infty$ and $N_{A}\left(\varrho_{2}\right)<+\infty$. On the other hand if these latter finiteness conditions are not satisfied the affine property follows from the definition of $N_{A}$.

Thus one sees that the study of the domain of the affine lower semicontinuous function $N_{A}$, i.e. the set of states $\varrho$ such that $N_{A}(\varrho)<+\infty$, is equivalent to the study of the set of states $\varrho$ which generate representa.tions with number operators $N_{\varrho}$ such that $\varrho$ can be extended to $N_{\varrho}$. This characterization is technically of use because the study of affine functionals with continuity properties is less complicated than the study of unbounded operators in Hilbert space.

The above theorem gives properties of $N_{A}$ as a function over the states $\varrho$ of $\mathscr{U}(A)$; next we wish to examine the function $A \subset R^{v} \rightarrow N_{A}(0)$ with $\varrho$ fixed. This is a straightforward study which we make as a preliminary to the introduction of a mean density functional over the set of translationally invariant states of $\mathfrak{A}$.

Lemma 1. Let $\Lambda, A^{\prime}$ be bounded open sets in $R^{v}$. If $A \subset A^{\prime}$ then

$$
N_{\Lambda^{\prime}}(\varrho) \geqq N_{\Lambda}(\varrho) \geqq 0, \quad \varrho \in E_{\mathscr{U}\left(A^{\prime}\right)}
$$

if however $A \cap A^{\prime}=0$ then

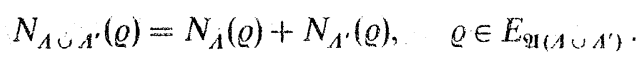


Finally if $\varrho \in E \cap L_{R^{v}}^{\perp}$ is a translationally invariant state over $\mathfrak{A}$ then

$$
N_{A}(\varrho)=N_{A+a}(\varrho), \quad a \in R^{v} .
$$

Proof. Let $\mathscr{F}_{\Lambda}=\left\{f_{A}\right\}$ and $\mathscr{F}_{\Lambda^{\prime} / \Lambda}=\left\{f_{\Lambda^{\prime} / \Lambda}\right\}$ be two finite families of orthonormal functions in $L^{2}(\Lambda)$ and $L^{2}\left(\Lambda^{\prime} / \Lambda\right)$ respectively then

$$
\begin{gathered}
\sum_{f_{\Lambda} \in \mathscr{F}_{\Lambda}} \varrho\left(a\left(f_{\Lambda}\right)^{*} a\left(f_{\Lambda}\right)\right)+\sum_{f_{\Lambda^{\prime} / \Lambda} \in \mathscr{F}_{\Lambda^{\prime} / A}} \varrho\left(a\left(f_{\Lambda^{\prime} / A}\right)^{*} a\left(f_{\Lambda^{\prime} / A}\right)\right) \\
\geqq \sum_{f_{\Lambda} \in \mathscr{F}_{A}} \varrho\left(a\left(f_{\Lambda}\right)^{*} a\left(f_{\Lambda}\right)\right) \geqq 0 .
\end{gathered}
$$

But the family $\left\{f_{\Lambda^{\prime}}, f_{\Lambda^{\prime} / \Lambda}\right\}$ forms a finite family of orthonormal functions in $L^{2}\left(\Lambda^{\prime}\right)$. Thus taking supremums over the possible families $\mathscr{F}_{A}$ and $\mathscr{F}_{A^{\prime} / \Lambda}$ we find the first statement of the lemma. To deduce the second statement we note that it is sufficient to assume

$$
N_{\Lambda}(\varrho)<+\infty, \quad N_{A^{\prime}}(\varrho)<+\infty
$$

because in the contrary case the statement is a consequence of the first result. However under these assumptions we know from Condition 3 of Theorem 1 that

$$
N_{\Lambda}(\varrho)+N_{A^{\prime}}(\varrho)=\operatorname{Lim}_{N \rightarrow \infty} \sum_{i=1}^{N} \varrho\left(a\left(f_{\Lambda}^{i}\right)^{*} a\left(f_{\Lambda}^{i}\right)+a\left(f_{\Lambda^{\prime}}^{i}\right)^{*} a\left(f_{A^{\prime}}^{i}\right)\right)<+\infty
$$

where $\left\{f_{A}^{i}\right\}_{i \geqq 1}$ and $\left\{f_{A^{\prime}}^{i}\right\}_{i \geqq 1}$ are any two complete orthonormal basis in $L^{2}(\Lambda)$ and $L^{2}\left(\Lambda^{\prime}\right)$ respectively. But the family $\left\{f_{\Lambda}^{i}, f_{\Lambda^{\prime}}^{i}\right\}_{i, j \geqq 1}$ forms a complete orthonormal basis in $L^{2}\left(\Lambda \cup \Lambda^{\prime}\right)$ and hence the result follows from Theorem 1. The invariance property is an immediate consequence of the action of the group of space translations.

Thus we see that $A \rightarrow N_{A}(\varrho)$ is a positive, increasing, additive function over the open bounded sets $\Lambda \subset R^{v}$. One may also remark that if $\Lambda=0$, the empty set, then $N_{\Lambda}(\varrho)=0$. Further if $\varrho$ is a translationally invariant state over $\mathfrak{U}$ then $A \rightarrow N_{A}(\varrho)$ is a translationally invariant function. These properties are sufficient to deduce that if $\varrho$ is invariant then $N_{\Lambda}(\varrho)$ is proportional to the Lebesgue measure (volume) $V(\Lambda)$ of $\Lambda$. The coefficient of proportionality introduced in this manner can then be considered as a functional over the invariant states; physically this functional corresponds to the mean density. This latter functional is of course of primary physical importance and we now study its properties.

Theorem 2. The mean density functional $N$, defined over the set $E \cap L_{R^{v}}^{\perp}$ of translationally invariant states over $\mathfrak{A}$ by

$$
\varrho \rightarrow E \cap L_{R^{v}}^{\perp} \rightarrow N(\varrho)=\frac{N_{\Lambda}(\varrho)}{V(\Lambda)}
$$


is independent of the open bounded set $\Lambda \subset R^{v}$ occurring in its definition. $N$ has the following properties:

0. $N(\varrho) \in[0,+\infty]$.

1. $N$ is affine i.e. if $\varrho_{1}, \varrho_{2} \in E \cap L_{R^{v}}^{\perp}$ and $0 \leqq \lambda \leqq 1$ then

$$
N\left(\lambda \varrho_{1}+(1-\lambda) \varrho_{2}\right)=\lambda N\left(\varrho_{1}\right)+(1-\lambda) N\left(\varrho_{2}\right) .
$$

2. $N$ is lower semi-continuous.

3. If the domain $F_{N}$ of $N$ is defined by

$$
F_{N}=\left\{\varrho ; \varrho \in E \cap L_{R^{v}}^{\perp}, N(\varrho)<+\infty\right\}
$$

then $\varrho \in F_{N}\left(=E \cap L_{\mathbb{R}^{\nu}}^{\perp} \cap F_{N}\right)$ implies that $\varrho$ is locally normal.

4. If $\varrho \in E \cap L_{R^{v}}^{\perp}$ and $\mu_{\varrho}$ is the unique probability measure with barycentre @ concentrated on $\mathscr{E}\left(E \cap L_{R^{\nu}}^{\perp}\right)$ then

$$
N(\varrho)=\int d \mu_{\varrho}\left(\varrho^{\prime}\right) N\left(\varrho^{\prime}\right)
$$

and if $\varrho \in F_{N}\left(=E \cap L_{R^{v}}^{\perp} \cap F_{N}\right)$ then $\mu_{o}$ is concentrated on $\mathscr{E}\left(E \cap L_{R^{v}}^{\perp}\right) \cap F_{N}$.

5. If $\varrho_{1}, \varrho_{2} \in \mathscr{E}\left(E \cap L_{R^{v}}^{\perp}\right) \cap F_{N}$ and $N\left(\varrho_{1}\right) \neq N\left(\varrho_{2}\right)$ then the representations $\pi_{\varrho_{1}}, \pi_{\varrho_{2}}$, generated by $\varrho_{1}$ and $\varrho_{2}$ respectively, are quasi-inequivalent.

Proof. The fact that the definition of $N$ is independent of $A$ is a staightforward exercise in measure theory which utilises the properties of the function $\Lambda \rightarrow N_{\Lambda}(\varrho)$ which we have derived above. We omit the details. The first four properties of $N$ are an immediate consequence of the analogous properties of $N_{A}$ derived in Theorem 1. The existence of the integral representation of $N$ follows from Properties 1 and 2 and the proof of Lemma 10 in [13]. We now prove that if $\varrho \in E \cap L_{R^{\nu}}^{\perp} \cap F_{N}$ then $\mu_{\varrho}$ is concentrated on $\mathscr{E}\left(E \cap L_{R^{v}}^{\perp}\right) \cap F_{N}$.

Define the set $G_{M}$ by

$$
G_{M}=\left\{\varrho ; \varrho \in \mathscr{E}\left(E \cap L_{R^{v}}^{\perp}\right), N(\varrho)>M\right\}
$$

and let $\mu_{o}=\mu^{\prime}+\mu^{\prime \prime}$ where, $\left\|\mu^{\prime}\right\|+\left\|\mu^{\prime \prime}\right\|=1, \mu^{\prime}$ is carried by $G_{M}$, and $\mu^{\prime \prime}$ by $\mathscr{E}\left(E \cap L_{R^{v}}^{\perp}\right) / G_{M}$. Then for $\varrho \in F_{N}$ we have

$$
+\infty>N(\varrho)=\mu_{\varrho}(N) \geqq \mu^{\prime}(N) \geqq M\left\|\mu^{\prime}\right\| .
$$

Thus $\left\|\mu^{\prime}\right\| \leqq N(\varrho) / M$ and hence $\mu_{\varrho}$ must be concentrated on $\mathscr{E}\left(E \cap L_{R^{v}}^{\perp}\right) \cap F_{N}$.

It remains to prove Property 5. The proof rests upon the following property [2]; $\varrho_{1}, \varrho_{2} \in \mathscr{E}\left(E \cap L_{R^{v}}^{\perp}\right)$ generate quasi-equivalent representations if, and only if, $\varrho_{1}=\varrho_{2}$. But now if

$$
N\left(\varrho_{1}\right)<N\left(\varrho_{2}\right)<+\infty
$$


then by Condition 3 of Theorem 1 we can choose a basis $\left\{f_{\Lambda}^{i}\right\}_{i \geqq 1}$, of orthonormal functions in $L^{2}(\Lambda)$ and an integer $N_{0}$ such that

$$
\sum_{i=1}^{N_{0}} \varrho_{1}\left(a\left(f_{\Lambda}^{i}\right)^{*} a\left(f_{\Lambda}^{i}\right)\right) \leqq N\left(\varrho_{1}\right) V(\Lambda)<\sum_{i=1}^{N_{0}} \varrho_{2}\left(a\left(f_{\Lambda}^{i}\right)^{*} a\left(f_{\Lambda}^{i}\right)\right) \leqq N\left(\varrho_{2}\right) V(\Lambda) .
$$

Hence $\varrho_{1} \neq \varrho_{2}$ and the proof is complete.

It is often technically disadvantageous that neither the set of invariant locally normal states nor the set of invariant states with finite density is weakly compact. However as a result of the lower semi-continuity of $N$ we can deduce the following.

Corollary 1. The set $F_{N}^{n}$ defined by

$$
F_{N}^{n}=\left\{\varrho ; \varrho \in E \cap L_{R^{\nu}}^{\perp} N(\varrho) \leqq n\right\}
$$

is convex and weakly compact.

The set is obviously convex but due to the semi-continuity it is closed. As $E \cap L_{R^{v}}^{\perp}$ is weakly compact it follows that $F_{N}^{n}$ is also weakly compact.

Note that one could have drawn a similar conclusion concerning states over the local algebras $\mathfrak{A}(\Lambda)$.

The above theorem characterizes the properties of states with finite mean density. Note that from Property 4 it follows that the barycentric decomposition of invariant states respects the property of finite mean density or, equivalently, the property of local normality given in Theorem 1. This is a weaker form of the result of Ruelle [3] which we recalled in Proposition 2.

In Theorem 1 we have seen that there exists a local number operator associated with each state of finite local density. It is natural to ask whether one can associate an unbounded density operator with each state of finite mean density. Our next aim is to demonstrate that this is indeed the case and thereby obtain a more explicit explanation of the decomposition properties of states with finite mean density and a characterization of ergodic states which share this property.

Theorem 3. Let $\varrho \in E \cap L_{R^{\nu}}^{\perp} \cap F_{N}$ be an invariant state with finite mean density $N(\varrho)$ and let $\left(\pi_{\varrho}, U_{\varrho}, \mathscr{H}_{\varrho}, \Omega_{\varrho}\right)$ be the representation of $\mathfrak{A}$, the unitary representation of $R^{v}$, the Hilbert space, and cyclic invariant vector, associated with @ by the Gelfand-Segal construction.

There exists a positive self-adjoint operator $N_{\varrho}$ on $\mathscr{H}_{Q}$ with the following properties:

1. $N(\varrho)=\left(\Omega_{\varrho}, N_{\varrho} \Omega_{\varrho}\right)$ and in particular $\Omega_{\varrho} \in \mathscr{D}\left(N_{\varrho}^{\frac{1}{2}}\right)$, the domain of $N_{\varrho}^{\frac{1}{2}}$.

2. The following commutation relations are valid

$$
\begin{array}{ll}
{\left[N_{\varrho}, \pi_{\varrho}(A)\right]=0,} & A \in \mathfrak{A} \\
{\left[N_{\varrho}, U_{\varrho}(x)\right]=0,} & x \in R^{v}
\end{array}
$$


and in particular, $\pi_{\varrho}$ and $U_{Q}$ leave the domain of $N_{Q}$ invariant. Hence $N_{Q}$ is affiliated with the abelian algebra $\left\{\pi_{\rho}, U_{e}\right\}^{\prime}$ of operators on $\mathscr{H}_{e}$.

3. If $\varrho \in \mathscr{E}\left(E \cap L_{R^{v}}^{\perp}\right) \cap F_{N}$ then $N_{\varrho}=N(\varrho) 1_{\varrho}$ where $1_{\varrho}$ is the identity operator on $\mathscr{H}_{Q}$.

Proof. The proof relies upon the combination of the techniques developed above and the Hilbert space techniques developed previously for the discussion of invariant states [2].

The major point that has to be demonstrated is the following. For each $A \in \mathfrak{A}$ let $\varrho_{A}$ be the vector state associated with $\varrho \in E \cap L_{R^{v}}^{\perp}$ by the definition

$$
\varrho_{A}(B)=\varrho\left(A^{*} B A\right) \quad B \in \mathfrak{Q} .
$$

Further define the parallelepiped $\Lambda_{l}$ by

$$
\Lambda_{l}=\left\{x ; x \in R^{v},-l_{i}<x_{i}<l_{i}, i=1, \ldots v\right\} .
$$

We wish to prove that if $N(\varrho)<+\infty$ then the limit

$$
N\left(\varrho_{A}\right)=\operatorname{Lim}_{l_{1} \ldots l_{v} \rightarrow \infty} \frac{N_{A_{1}}\left(\varrho_{A}\right)}{V\left(\Lambda_{l}\right)}
$$

exists for a dense set of $A \in \mathfrak{A}$ and is invariant in the sense that

$$
N\left(\varrho_{A}\right)=N\left(\varrho_{\tau_{x} A}\right), \quad x \in R^{v} .
$$

Once we have done this we will construct a self-adjoint operator $N_{\varrho}$ on $\mathscr{H}_{Q}$ such that

$$
N\left(\varrho_{A}\right)=\left(\pi_{\varrho}(A) \Omega_{\varrho}, N_{\varrho} \pi_{\varrho}(A) \Omega_{\varrho}\right)
$$

and demonstrate that this operator has all the properties stated in the theorem.

Let $n_{i}, i=1, \ldots v$ be positive integers and denote by $n l$ the $v$-vector $\left(n_{1} l_{1}, \ldots, n_{v} l_{v}\right)$. We first show that if the limit

$$
N_{l}\left(\varrho_{A}\right)=\operatorname{Lim}_{n_{1}, \ldots n_{v} \rightarrow \infty} \frac{N_{A_{n} l}\left(\varrho_{A}\right)}{V\left(\Lambda_{n l}\right)}
$$

exists then it is independent of $l_{1}, \ldots, l_{v}$. To deduce this we introduce vectors $l_{1}=\left(l_{11}, \ldots, l_{1 v}\right), l_{2}=\left(l_{21}, \ldots, l_{2 v}\right)$ and note that for a given value of $n_{i}$ it is possible to choose an integer $m_{i}$ such that

$$
m_{i} l_{2 i} \leqq n_{i} l_{1 i}<\left(m_{i}+1\right) l_{2 i} .
$$

Hence applying the increase property of $N_{A}(\varrho)$ deduced in Lemma 1 to the state $\varrho_{A}$ where $A$ is assumed strictly local we find

$$
\frac{V\left(\Lambda_{m l_{2}}\right)}{V\left(\Lambda_{m l_{1}}\right)} \frac{N_{\Lambda_{m l_{2}}}\left(\varrho_{A}\right)}{V\left(\Lambda_{m l_{2}}\right)} \leqq \frac{N_{\Lambda_{m l_{1}}}\left(\varrho_{A}\right)}{V\left(\Lambda_{m l_{1}}\right)} \leqq \frac{N_{\Lambda_{(m+1) l_{2}}}\left(\varrho_{A}\right)}{V\left(\Lambda_{(m+1) l_{2}}\right)} \frac{V\left(\Lambda_{(m+1) l_{2}}\right)}{V\left(\Lambda_{m l_{1}}\right)}
$$


In the limit $n_{1}, \ldots, n_{v} \rightarrow \infty$ we have $m_{1}, \ldots, m_{v} \rightarrow \infty$ and hence

$$
N_{l_{2}}\left(\varrho_{A}\right)=N_{l_{1}}\left(\varrho_{A}\right)=N\left(\varrho_{A}\right) \text {. }
$$

A similar argument based on the increase property and the transformation law

$$
N_{A}\left(\varrho_{\tau_{x} A}\right)=N_{A-x}\left(\varrho_{A}\right)
$$

establishes that if $N_{l}\left(\varrho_{A}\right)$ exists then

$$
N\left(\varrho_{A}\right)=N_{l}\left(\varrho_{A}\right)=N_{l}\left(\varrho_{\tau_{x} A}\right)=N\left(\varrho_{\tau_{x} A}\right), \quad x \in R^{v} .
$$

We will not elaborate on this point but turn our attention to proving the existence of $N_{l}\left(\varrho_{A}\right)$ for a suitably chosen set of $A \in \mathfrak{A}$.

Let $\mathscr{F}_{A_{l}}=\left\{\mathrm{f}_{A_{l}}^{i}\right\}_{i \geqq 1}$ be a complete orthonormal basis in $L^{2}\left(\Lambda_{l}\right)$. Under the assumption $N(\varrho)<+\infty$ Theorem 1 tells us that

$$
N_{\Lambda_{l}}(\varrho)=\operatorname{Lim}_{N \rightarrow \infty} \sum_{i=1}^{N}\left(\Omega_{\varrho}, \pi_{\varrho}\left(a\left(f_{A_{l}}^{i}\right)^{*} a\left(f_{\Lambda_{l}}^{i}\right)\right) \Omega_{\varrho}\right)
$$

and hence for $\varepsilon>0$ one can choose $N_{0}$ such that

$$
\left|N_{\Lambda_{l}}(\varrho)-\sum_{i=1}^{N}\left(\Omega_{\varrho}, \pi_{\varrho}\left(a\left(f_{\Lambda_{l}}^{i}\right)^{*} a\left(f_{\Lambda_{l}}^{i}\right)\right) \Omega_{\varrho}\right)\right|<\varepsilon \text { for } N>N_{0} .
$$

However if $A \in \mathfrak{A}\left(\Lambda_{l}\right)$ is a polynomial in the annihilation and creation operators $a\left(f_{A_{l}}^{i}\right), a\left(f_{A_{l}}^{i}\right)^{*}$ then we can choose $N_{0}$ such that

$$
\begin{aligned}
0 & \leqq \sum_{i=N_{1}}^{N_{2}}\left(\Omega_{\varrho}, \pi_{\varrho}\left(A^{*} a\left(f_{\Lambda_{l}}^{i}\right)^{*} a\left(f_{\Lambda_{l}}^{i}\right) A\right) \Omega_{\varrho}\right) \\
& \left.\leqq\|A\|^{2} \sum_{i=N_{1}}^{N_{2}}\left(\Omega_{\varrho}, \pi_{\varrho}\left(a\left(f_{\Lambda_{l}}^{i}\right)^{*} a\left(f_{\Lambda_{l}}^{i}\right)\right)\right) \Omega_{\varrho}\right)
\end{aligned}
$$

for all $N_{2} \geqq N_{1}>N_{0}$. The last statement follows from the same calculation used in proving the equivalence of Conditions 3 and 4 in Proposition 1; it is a consequence of the anti-commutation relations. Thus for $\varepsilon>0$ we can choose $N_{0}$ such that the above estimate and the estimate

$$
\left|N_{\Lambda_{l}}\left(\varrho_{A}\right)-\sum_{i=1}^{N}\left(\Omega_{\varrho}, \pi_{\varrho}\left(A^{*} a\left(f_{\Lambda_{l}}^{i}\right)^{*} a\left(f_{\Lambda_{l}}^{i}\right) A\right) \Omega_{\varrho}\right)\right|<\varepsilon \text { for } N>N_{0}
$$

are simultaneously valid. Note that if $x \in R^{v}$ is such that $\left(\Lambda_{l}+x\right) \cap \Lambda_{l}=0$ then it also follows that

$$
\left|N_{\Lambda_{l}}\left(\varrho_{\tau_{x} A}\right)-\sum_{i=1}^{N}\left(\Omega_{\varrho}, \pi_{\varrho}\left(\tau_{x} A^{*} a\left(f_{A_{l}}^{i}\right)^{*} a\left(f_{\Lambda_{l}}^{i}\right) \tau_{x} A\right) \Omega_{\varrho}\right)\right|<\varepsilon \text { for } N>N_{0} .
$$

This latter uniformity criterion is essential in the following proof. 
Introduce the set $\theta_{n} \subset Z^{v}$ by the definition

$$
\theta_{n}=\left\{m ; m \in Z^{v},-n_{i} \leqq m_{i} \leqq n_{i}\right\}
$$

and let $N\left(\theta_{n}\right)$ denote the number of points in $\theta_{n}$ then from the additivity and transformation properties of $N_{A}\left(\varrho_{A}\right)$ we have

$$
\frac{N_{\Lambda_{(2 n+1) l}}\left(\varrho_{A}\right)}{V\left(\Lambda_{(2 n+1) l}\right)}=\frac{1}{N\left(\theta_{n}\right)} \sum_{m \in \theta_{n}} \frac{N_{\Lambda_{l}}\left(\varrho_{\tau_{m l} A}\right)}{V\left(\Lambda_{l}\right)} .
$$

Hence combining this formula and the estimates given above

$$
\begin{aligned}
& \mid \frac{N_{\Lambda_{(2 n+1) l}}\left(\varrho_{A}\right)}{V\left(\Lambda_{(2 n+1) l}\right)}- \frac{1}{V\left(\Lambda_{l}\right)} \frac{1}{N\left(\theta_{n}\right)} \\
& \cdot \sum_{m \in \theta_{n}}\left(\Omega_{\varrho}, \pi_{\varrho}\left(A^{*}\right) \sum_{i=1}^{N} \pi_{\varrho}\left(\tau_{m l}\left(a\left(f_{\Lambda_{l}}^{i}\right)^{*} a\left(f_{\Lambda_{l}}^{i}\right)\right)\right) \pi_{\varrho}(A) \Omega_{\varrho}\right) \mid<\varepsilon \\
& \text { for all } \quad N>N_{0} .
\end{aligned}
$$

Next let us introduce the notation

$$
A_{N, l}=\frac{1}{V\left(\Lambda_{l}\right)} \sum_{i=1}^{N} a\left(f_{\Lambda}^{i}\right)^{*} a\left(f_{\Lambda}^{i}\right) .
$$

Then, noting that $A_{N, l} \in \mathfrak{A}$, we have from [2] that the limit

$$
\begin{aligned}
\lim _{n_{1} \ldots n_{v} \rightarrow \infty} \frac{1}{N\left(\theta_{n}\right)} \sum_{m \in \theta_{n}}\left(\pi_{\varrho}(A) \Omega_{\varrho}, \pi_{\varrho}\left(\tau_{m l} A_{N, l}\right) \pi_{\varrho}(A) \Omega_{\varrho}\right) \\
=\left(\pi_{\varrho}(A) \Omega_{\varrho}, M\left(A_{N, l}\right) \pi(A) \Omega_{\varrho}\right)
\end{aligned}
$$

exists and defines a bounded operator $M\left(A_{N, l}\right)$ whose action is such that

$$
\left(\pi_{\varrho}(A) \Omega_{\varrho}, M\left(A_{N, l}\right) \pi_{\varrho}(A) \Omega_{\varrho}\right)=\left(\Omega_{\varrho}, \pi_{\varrho}\left(A^{*} A\right) E_{\varrho}^{l} \pi_{\varrho}\left(A_{N l}\right) \Omega_{\varrho}\right)
$$

where $E_{\varrho}^{l}$ is the projector on the subspace of $\mathscr{H}_{\varrho}$ spanned by all vectors $\Psi$ such that

$$
U_{\varrho}(m l) \Psi=\Psi, \quad m \in Z^{\nu} .
$$

Combining the above results we immediately have the existence of $N\left(\varrho_{A}\right)$ and in fact

$$
\begin{aligned}
N\left(\varrho_{A}\right) & =\lim _{N \rightarrow \infty}\left(\pi_{\varrho}(A) \Omega_{\varrho}, M\left(A_{N l}\right) \pi_{\varrho}(A) \Omega_{\varrho}\right) \\
& =\lim _{N \rightarrow \infty}\left(\Omega_{\varrho}, \pi_{\varrho}\left(A^{*} A\right) E_{\varrho}^{l} \pi_{\varrho}\left(A_{N, l}\right) \Omega_{\varrho}\right) \\
& =\left(\Omega_{\varrho}, \pi_{\varrho}\left(A^{*} A\right) E_{\varrho}^{l} \frac{N_{\Lambda_{l}}}{V\left(\Lambda_{l}\right)} \Omega_{\varrho}\right)
\end{aligned}
$$

where the last statement follows from the characterization of the number operator given in Section 2 and the results of Theorem $1\left(N_{A_{l}}\right.$ denotes the 
number operator which is associated with $\pi_{\varrho}$ by virtue of Theorem 1). But having deduced the existence of $N(\varrho)$ we then know from the discussion at the beginning of the proof that

and hence

$$
N\left(\varrho_{A}\right)=N\left(\varrho_{\tau_{x} A}\right), \quad x \in R^{v}
$$

$$
N\left(\varrho_{A}\right)=\left(\Omega_{\varrho}, \pi_{\varrho}\left(A^{*} A\right) E_{\varrho} \frac{N_{\Lambda_{l}}}{V\left(\Lambda_{l}\right)} \Omega_{\varrho}\right)
$$

where $E_{\varrho}$ is the projector on the subspace of $\mathscr{H}_{\varrho}$ spanned by the $R^{v}$ invariant vectors i.e. the vectors $\Psi$ such that

$$
U_{\varrho}(x) \Psi=\Psi, \quad x \in R^{v} .
$$

The introduction of the self adjoint operator $N_{\varrho}$ is now made in the following manner. The operators $M\left(A_{N, l}\right)$ form an increasing sequence of positive bounded operators and we have deduced above that

$$
\lim _{N \rightarrow \infty}\left(\pi_{\varrho}\left(A^{*}\right) \Omega_{\varrho} M\left(A_{N l}\right) \pi_{\varrho}(A) \Omega_{\varrho}\right)=\left(\Omega_{\varrho}, \pi_{\varrho}\left(A^{*} A\right) E_{\varrho} \frac{N_{\Lambda_{l}}}{V\left(\Lambda_{l}\right)} \Omega_{\varrho}\right)<+\infty
$$

for a dense set of $A \in \mathfrak{A}$. Hence from [5] there exists a positive self adjoint operator $N_{\varrho}$ on $\mathscr{H}_{\varrho}$ which is such that

$$
\begin{aligned}
\left(\pi_{\varrho}\left(A^{*}\right) \Omega_{\varrho}, N_{\varrho} \pi_{\varrho}(A) \Omega_{\varrho}\right) & =\lim _{N \rightarrow \infty}\left(\pi_{\varrho}\left(A^{*}\right) \Omega_{\varrho}, M\left(A_{N, l}\right) \pi_{\varrho}(A) \Omega_{\varrho}\right) \\
& =\left(\Omega_{\varrho}, \pi_{\varrho}\left(A^{*} A\right) E_{\varrho} \frac{N_{\Lambda_{l}}}{V\left(\Lambda_{l}\right)} \Omega_{\varrho}\right)
\end{aligned}
$$

whenever the limit exists and whose other matrix elements are obtained by polarization and closure of the bilinear form introduced by the limit. All stated properties of $N_{\varrho}$ follow immediately from the above characterization, Proposition 2, and the characterization of extremal invariant states [2].

We remark that the existence of $N_{\varrho}$ and its affiliation with the abelian algebra $\left\{\pi_{\varrho}, U_{\varrho}\right\}^{\prime}$ explains essentially the reason why the barycentric decomposition of invariant states respects the property of finite mean density. This decomposition is induced by decomposition of the representation $\pi_{e}$ obtained by "diagonalization" of the central subalgebra $\left\{\pi_{\varrho}, U_{\varrho}\right\}^{\prime}$ (see [2]) and of course any affiliated unbounded operators. The ergodicity of $\varrho$ is equivalent to the triviality of $\left\{\pi_{\varrho}, U_{\varrho}\right\}^{\prime}$ and this is why ergodicity implies that $N_{\varrho}$ is a multiple of the identity.

Physically the criterion of ergodicity $\varrho \in \mathscr{E}\left(E \cap L_{R^{v}}^{\perp}\right)$ corresponds to the absence of large fluctuations in the space averages of local observables. In this light the requirement $\varrho \in \mathscr{E}\left(E \cap L_{R^{v}}^{\perp}\right) \cap F_{N}$ corresponds to the consideration of states with small density fluctuations. This qualitative 
property is however a little difficult to formulate quantitatively because for $\varrho \in F_{N}$ it does not necessarily follow that $\Omega_{\varrho}$ is in the domain of the unbounded number operators $N_{A}$.

\section{Kinetic Energy and Mean Kinetic Energy}

Our next aim is to analyse the states over $\mathfrak{A}$ which can be physically interpreted to have finite mean kinetic energy and finite particle density. Our analysis follows the same general pattern as that developed in the previous section.

We begin by considering the kinetic energy of a finite system and again introduce this concept by reference to the Fock representation $\pi_{A}$ of $\mathfrak{U}(\Lambda)$. Let $\mathscr{D}_{0}(\Lambda)$ be the dense subspace of the Fock space $\mathscr{H}_{F}(\Lambda)$ spanned by twice continuously differentiable functions vanishing on the boundaries of $\Lambda$. On $\mathscr{D}_{0}(\Lambda)$ we can define a positive symmetric operator $T_{A}^{0}$ by

$$
\left(T_{A}^{0} \Psi\right)^{(n)}\left(x_{1}, \ldots x_{n}\right)=-\sum_{i=1}^{n} \nabla_{x_{i}}^{2} \Psi^{(n)}\left(x_{1}, \ldots x_{n}\right)
$$

where $\nabla_{x}^{2}$ is the Laplace operator. Physically one expects the kinetic energy operator to be given as a self adjoint extension of $T_{\Lambda}^{0}$. At this point however a certain ambiguity arises; $T_{A}^{0}$ is not essentially self adjoint but has many different self adjoint extensions. These different extensions arise from different choices of boundary conditions. We will circumvent this set of ambiguities by choosing one particular self adjoint extension $T_{A}$ of $T_{A}^{0}$ which is particularly suited to our present purpose. Note that insofar we derive properties of states of infinite systems these should be independent of the particular self adjoint extension chosen for the description of finite subsystems; however we have not explicitly verified this independence.

We now proceed to give various characterizations of the kinetic energy operator $T_{A}$. Let $\mathscr{F}=\left\{f_{A}\right\}$ be a complete orthonormal basis of $L^{2}(\Lambda)$ formed by once continuously differentiable functions $f_{A}$ which vanish on the boundaries of $\Lambda$. Define the set $\mathscr{D}(\Lambda)$ by

$$
\mathscr{D}(\Lambda)=\left\{\Psi ; \Psi \in \mathscr{H}_{F}(\Lambda), \sup _{\mathscr{F}} \sum_{f_{\Lambda} \in \mathscr{F}}\left(\Psi, \pi_{\Lambda}\left(a\left(\nabla f_{A}\right) * a\left(\nabla f_{A}\right)\right) \Psi\right)<+\infty\right\} .
$$

The proofs of [5] establish the existence of a self adjoint operator $T_{A}$ with the property that

$$
\left(\Psi, T_{\Lambda} \Psi\right)=\sup _{\mathscr{F}} \sum_{f_{A} \in \mathscr{F}}\left(\Psi, \pi_{\Lambda}\left(a\left(\nabla f_{\Lambda}\right)^{*} a\left(\nabla f_{\Lambda}\right)\right) \Psi\right)
$$


for all $\Psi \in \mathscr{D}(\Lambda)$ and further show that $\mathscr{D}(\Lambda)=\mathscr{D}\left(T_{\hat{A}}^{\frac{1}{2}}\right)$, the domain of $T_{\hat{A}}^{\frac{1}{2}}$. A straightforward computation involving partial integration and the completeness of $\mathscr{F}$ establishes that $T_{A}$ is an extension of $T_{A}^{0}$.

The abstract definition of $T_{A}$ given above is of great use but fails to give very detailed knowledge of the properties of $T_{A}$, e.g. it is not clear whether $T_{A}$ is independent of the basis $\mathscr{F}$ occurring in its definition. Next we argue that $T_{A}$ is the extension of $T_{A}^{0}$ whose eigenfunctions $\Psi^{(n)} \in \mathscr{H}_{F}(\Lambda)$ satisfy the differential equation

$$
-\sum_{i=1} \nabla_{x_{i}}^{2} \Psi^{(n)}\left(x_{1} \ldots x_{n}\right)=E_{n} \Psi^{(n)}\left(x_{1} \ldots x_{n}\right)
$$

with the boundary condition $\nabla_{x_{i}} \Psi^{(n)}\left(x_{1} \ldots x_{n}\right)=0$ across the boundary of $\Lambda$. Note that it is sufficient to establish this property on the one particle subspace of $\mathscr{H}_{F}(\Lambda)$ because one sees from the definition of $T_{A}$ that the multi particle eigenfunctions are formed by taking suitably antisymmetrized products of the one particle eigenfunctions. Now let $\Psi$ be a one particle eigenfunction of $T_{A}$ with eigenvalue $E$ and take $\Phi \in \mathscr{D}(\Lambda)$ then

$$
\begin{aligned}
E(\Phi, \Psi) & =\sum_{f_{\Lambda} \in \mathscr{F}}\left(\Phi, \nabla f_{\Lambda}\right) \cdot\left(\nabla f_{\Lambda}, \Psi\right) \\
& =(\nabla \Phi, \nabla \Psi) \\
& =-\left(\Phi, \nabla^{2} \Psi\right)+[\Phi \nabla \Psi]_{\text {bound }} \\
& =E(\Phi, \Psi)+[\Phi \nabla \Psi]_{\text {bound. }}
\end{aligned}
$$

The first step uses the action of $a(\nabla f)$ on the one particle subspace of $\mathscr{H}_{F}(\Lambda)$, the second step follows by partial integration and the completeness of $\mathscr{F}$. The third step involves another partial integration and introduces a surface term which we have denoted by $[\Phi \nabla \Psi]_{\text {bound }}$. For consistency this surface term must vanish for all $\Phi \in \mathscr{D}(\Lambda)$ regardless of the values of $\Phi$ on the boundary, i.e. $\nabla \Psi$ must vanish across the boundary. In particular, for $\Psi \in \mathscr{D}(\Lambda),\left(\Psi, T_{A} \Psi\right)$ is independent of the basis $\mathscr{F}$ used in its definition. Further if $\left\{f_{n}\right\}$ is the complete orthonormal basis of $L^{2}(\Lambda)$ formed by the one particle eigenfunctions of $T_{A}$ and $\varepsilon_{n}$ are the associated eigenvalues and the indices are ordered such that

then we have

$$
0=\varepsilon_{0}<\varepsilon_{1} \leqq \varepsilon_{2} \leqq \cdots
$$

$$
T_{\Lambda}=\sum \varepsilon_{n} \pi_{\Lambda}\left(a\left(f_{n}\right)^{*} a\left(f_{n}\right)\right) .
$$

This last formula allows us to deduce that

$$
T_{\Lambda} \geqq \varepsilon_{1} \sum_{n \geqq 1} \pi_{A}\left(a\left(f_{n}\right)^{*} a\left(f_{n}\right)\right) \geqq \varepsilon_{1}\left[N_{A}-1\right]
$$

i.e. $T_{A}$ dominates $N_{A}$ in the sense given. 
In the following section we will need certain estimates involving the eigenvalues of $T_{A}$ and these are most easily obtained by restricting $\Lambda$ to be a parallelepiped, the lengths of whose sides we denote by $l_{1}, \ldots l_{v}$. Then the one-particle eigenfunctions $C_{A}^{n}$ of $T_{A}$ are given by

$$
C_{\Lambda}^{n}(x)=\prod_{i=1}^{v} a_{i} \operatorname{Cos} \frac{n_{i} x_{i}}{l_{i}} \pi, \quad x=\left(x_{1}, \ldots x_{v}\right), 0 \leqq x_{i} \leqq l_{i}
$$

and $n=\left(n_{1}, \ldots n_{v}\right)$ where the $n_{i}$ are non-negative integers; the normalization constants $a_{i}$ are given by $a_{i}=\sqrt{1 / l_{i}}$ if $n_{i}=0$ and $a_{i}=\sqrt{2 / l_{i}}$ if $n_{i}>0$.

The associated eigenvalues $\varepsilon_{n}$ are given by

and we have for $\beta>0$

$$
\varepsilon_{n}=\sum_{i=1}^{v}\left(\frac{\pi n_{i}}{l_{i}}\right)^{2}
$$

$$
\begin{aligned}
\operatorname{Tr}_{\mathscr{H}_{F}(\Lambda)}\left(e^{-\beta T_{A}}\right) & =\prod_{n}\left(1+e^{-\beta \varepsilon_{n}}\right) \\
& \leqq \exp \left\{\sum_{n} e^{-\beta \varepsilon_{n}}\right\} \\
& \leqq \exp \left\{\prod_{i=1}^{v}\left(1+\int_{0}^{\infty} d x \exp \left\{-\beta \pi^{2} x^{2} / l_{i}^{2}\right)\right\}\right. \\
& =\exp \left\{\frac{V(\Lambda)}{(4 \pi \beta)^{v / 2}} \prod_{i=1}^{v}\left(1+\frac{\sqrt{4 \pi \beta}}{l_{i}}\right)\right\} .
\end{aligned}
$$

Motivated by the above characterization of the kinetic energy operator $T_{A}$ we now introduce the concept of a kinetic energy functional in a manner analogous to that previously adopted for the number functional.

Definition 2. Let $\mathscr{F}=\left\{f_{A}\right\}$ be a finite family of orthonormal once continuously differentiable functions $f_{\Lambda} \in L^{2}(\Lambda)$ which vanish on the boundaries of $\Lambda$. The kinetic energy functional $T_{A}$ is defined as a function from the states $E_{\mathfrak{U}(\Lambda)}$ of $\mathfrak{A}(\Lambda)$ to the interval $[0,+\infty]$ by

$$
T_{\Lambda}(\varrho)=\sup _{\mathscr{F}} \sum_{f_{A} \in \mathscr{F}} \varrho\left(a\left(\nabla f_{A}\right)^{*} \cdot a\left(\nabla f_{A}\right)\right) .
$$

This definition allows one to characterize the states with finite kinetic energy and especially those which also have finite density.

Theorem 4. $T_{A}$ is an affine lower semi-continuous function over the convex weakly compact set $E_{\mathfrak{U}(\Lambda)}$ of states over $\mathfrak{U}(\Lambda)$. The following con- 
ditions are equivalent

1. $T_{\Lambda}(\varrho)<+\infty$ and $N_{\Lambda}(\varrho)<+\infty$.

2. $\varrho$ is normal with respect to the Fock representation, i.e. is determined by a density matrix $\varrho_{\Lambda}$ on $\mathscr{H}_{F}(\Lambda)$, and

$$
\operatorname{Tr}_{\mathscr{H}_{F}(\Lambda)}\left(\varrho_{\Lambda} T_{\Lambda}\right)<+\infty
$$

where $T_{\Lambda}$ is the self adjoint kinetic energy operator associated with the Fock representation.

If these latter conditions are satisfied then

$$
T_{\Lambda}(\varrho)=\operatorname{Tr}_{\mathscr{H}_{F}(\Lambda)}\left(\varrho_{\Lambda} T_{\Lambda}\right)=\sum_{f_{\Lambda} \in \mathscr{F}} \varrho\left(a\left(\nabla f_{\Lambda}\right)^{*} \cdot a\left(\nabla f_{\Lambda}\right)\right)
$$

where $\mathscr{F}=\left\{f_{\Lambda}\right\}$ is any complete orthonormal basis in $L^{2}(\Lambda)$ formed by once continuously differentiable functions $f_{\Lambda}$ which vanish on the boundaries of $\Lambda$.

Proof. $T_{A}$ is defined as the supremum of a family of continuous functions and hence is lower semi-continuous. It is also evident that $T_{A}$ is convex but the following argument due to Lanford establishes that it is affine. Consider

$$
T_{\Lambda}(\varrho ; \mathscr{F})=\sum_{f_{\Lambda} \in \mathscr{F}} \varrho\left(a\left(\nabla f_{\Lambda}\right)^{*} \cdot a\left(\nabla f_{\Lambda}\right)\right) .
$$

The finite family $\mathscr{F}=\left\{f_{\Lambda}\right\}$ spans a subspace $S$ of $L^{2}(\Lambda)$. If we choose any basis $\mathscr{F}_{S}$ of $S$ again formed by once differentiable functions vanishing on the boundary of $\Lambda$ then a simple calculation involving a unitary transformation shows that

$$
T_{\Lambda}(\varrho ; \mathscr{F})=T_{\Lambda}\left(\varrho ; \mathscr{F}_{S}\right)
$$

i.e. $T_{\lambda}(\varrho ; \mathscr{F})$ depends only on the subspace $S$. Thus $T_{\Lambda}(\varrho)$ is actually the limit over the net of finite dimensional subspaces of $L^{2}(\Lambda)$. Hence $T_{\Lambda}$ is the limit of a family of affine functionals and is consequently affine.

Now $N_{\Lambda}(\varrho)<+\infty$ implies that $\varrho$ is normal with respect to the Fock representation due to Theorem 1 but then $T_{\Lambda}(\varrho)<+\infty$ implies that

$$
\begin{aligned}
+\infty>T_{\Lambda}(\varrho) & =\sup _{\mathscr{F}} \operatorname{Tr}_{\mathscr{H}_{F}(\Lambda)}\left(\varrho_{\Lambda} \sum_{f_{\Lambda} \in \mathscr{F}^{\prime}} \pi_{\Lambda}\left(a\left(\nabla f_{\Lambda}\right)^{*} \cdot a\left(\nabla f_{\Lambda}\right)\right)\right) \\
& =\operatorname{Tr}_{\mathscr{H}_{F}(\Lambda)}\left(\varrho_{\Lambda} T_{\Lambda}\right)
\end{aligned}
$$

where the last step is a consequence of the above characterization of the kinetic energy operator $T_{A}$ and the monotone convergence theorem [12]. Hence Condition 1 implies Condition 2. Now we have seen that the kinetic energy operator $T_{A}$ dominates the number operator $N_{A}$ in the sense that there exists on $\varepsilon_{\Lambda}>0$ such that

$$
T_{\Lambda} \geqq \varepsilon_{\Lambda}\left[N_{\Lambda}-1\right] \text {. }
$$


Hence $+\infty>\operatorname{Tr}_{\mathscr{H}_{F}(\Lambda)}\left(\varrho_{A} T_{A}\right)$ implies $+\infty>\operatorname{Tr}_{\mathscr{H}_{F}}\left(\varrho_{A} N_{A}\right)$ and hence by Theorem 1 we have $N_{A}(\varrho)<+\infty$. $T_{A}(\varrho)<+\infty$ follows straightforwardly as a consequence of Condition 2 .

Before proceeding we remark that an inequality of the form

$$
T_{\Lambda}(\varrho) \geqq \varepsilon_{\Lambda}\left[N_{\Lambda}(\varrho)-1\right]
$$

is valid under the assumption that $\varrho$ is normal with respect to the Fock representation but it is possible that this assumption is not necessary. In particular in one dimension $(v=1)$ we have for the interval $\Lambda=(0, l)$

$$
\begin{aligned}
T_{\Lambda}(\varrho) & \geqq \sum_{n \geqq 1} \varrho\left(a\left(\nabla S_{\Lambda}^{n}\right)^{*} a\left(\nabla S_{\Lambda}^{n}\right)\right) \\
& =\sum_{n \geqq 1}\left(\frac{n \pi}{l}\right)^{2} \varrho\left(a\left(C_{\Lambda}^{n}\right)^{*} a\left(C_{A}^{n}\right)\right) \\
& \geqq\left(\frac{\pi}{l}\right)^{2}\left[N_{\Lambda}(\varrho)-1\right]
\end{aligned}
$$

where $S_{\Lambda}^{n}$ and $C_{A}^{n}$ are given by

$$
S^{n}(x)=\sqrt{2 / l} \sin \pi \frac{n x}{l}, \quad C^{n}(x)=\sqrt{2 / l} \cos \pi \frac{n x}{l}, \quad n=1,2, \ldots .
$$

If this inequality is in general valid then the statement of the theorem can be strengthened because $T_{\Lambda}(\varrho)<+\infty$ would imply $N_{\Lambda}(\varrho)<+\infty$.

Next we analyse the properties of the function $\Lambda \subset R^{v} \rightarrow T_{\Lambda}(\varrho)$ with $\varrho$ fixed.

Lemma 2. Let $\Lambda, \Lambda^{\prime}$ be bounded open sets in $R^{v}$. If $\Lambda \subset \Lambda^{\prime}$ then

$$
T_{A^{\prime}}(\varrho) \geqq T_{A}(\varrho) \geqq 0, \quad \varrho \in E_{\mathscr{Q}\left(\Lambda^{\prime}\right)} .
$$

If however $\Lambda \cap \Lambda^{\prime}=\theta$ then

$$
T_{A \cup A^{\prime}}(\varrho) \geqq T_{A^{\prime}}(\varrho)+T_{\Lambda}(\varrho), \quad \varrho \in E_{\mathfrak{Q}\left(A^{\prime} \cup \Lambda\right)}
$$

and the equality sign holds if $T_{A \cup A^{\prime}}(\varrho)<+\infty, N_{A \cup A^{\prime}}(\varrho)<+\infty$. Finally if $\varrho \in E \cap L_{R^{v}}^{\perp}$ is a translationally invariant state over $\mathfrak{A}$ then

$$
T_{\Lambda}(\varrho)=T_{A+a}(\varrho), \quad a \in R^{v} .
$$

Proof. The proof of the lemma is similar to that given in the previous section, Lemma 1 , for the function $\Lambda \rightarrow N_{\Lambda}(\varrho)$. The additivity of $T_{\Lambda}(\varrho)$ in the case stated above is a consequence of the monotone convergence theorem and the properties of the kinetic energy operator $T_{A}$; specifically we have for any complete orthonormal basis $\mathscr{F}=\left\{f_{A}\right\}$ in $L^{2}(\Lambda)$ of once continuously differentiable functions vanishing on the boundaries of $\Lambda$ that

$$
T_{\Lambda}(\varrho)=\sum_{f_{A} \in \mathscr{F}} \varrho\left(a\left(\nabla f_{A}\right)^{*} \cdot a\left(\nabla f_{A}\right)\right)=\operatorname{Tr}_{\mathscr{H}_{F}(\Lambda)}\left(\varrho_{A} T_{A}\right)
$$


whenever $T_{\Lambda}(\varrho)<+\infty$ and $N_{\Lambda}(\varrho)<+\infty$ due to theorem 4. Using the fact that the definition of $T_{\Lambda}(\varrho)$ is basis independent the additivity follows as in Lemma 1.

Before proceeding we remark that the additivity property derived in the Lemma implies that if $\varrho$ is a state of finite density and kinetic energy then

$$
\operatorname{Tr}_{\mathscr{H}_{F}\left(\Lambda \cup \Lambda^{\prime}\right)}\left(\varrho_{A \cup \Lambda^{\prime}} T_{A \cup A^{\prime}}\right)=\operatorname{Tr}_{\mathscr{H}_{F}(\Lambda)}\left(\varrho_{\Lambda} T_{\Lambda}\right)+\operatorname{Tr}_{\mathscr{H}_{F}\left(\Lambda^{\prime}\right)}\left(\varrho_{\Lambda^{\prime}} T_{\Lambda^{\prime}}\right)<+\infty .
$$

Now $T_{A}$ and $T_{A^{\prime}}$ can be considered, by extension, as operators on $\mathscr{H}_{F}\left(\Lambda \cup \Lambda^{\prime}\right)$ and this equality implies that

and

$$
\mathscr{D}\left(T_{\Lambda}^{\frac{1}{2}} \cup \Lambda^{\prime}\right) \subseteq \mathscr{D}\left(T_{\Lambda}^{\frac{1}{2}}\right) \cap \mathscr{D}\left(T_{\Lambda^{\prime}}^{\frac{1}{2}}\right)
$$

$$
\left\|T_{\lambda}^{\frac{1}{2}} \cup{A^{\prime}}^{\prime} \Psi\right\|^{2}=\left\|T_{\lambda}^{\frac{1}{3}} \Psi\right\|^{2}+\left\|T_{\hat{\lambda}^{\prime}}^{\frac{1}{2}}\right\|^{2}
$$

for $\Psi \in \mathscr{D}\left(T_{A}^{\frac{1}{2}} \cup A^{\prime}\right)$. However this in no way implies that the operator $T_{A}$ is additive, i.e. that $T_{A \cup A^{\prime}}=T_{A}+T_{A^{\prime}}$, and indeed this is not the case as one can deduce from counterexamples.

Secondly we note that this additivity property is fundamental for the extension of proofs of existence of thermodynamic functions to the case of the hard wall boundary conditions which we are using.

Combination of the above theorem and lemma now leads to the following result concerning the mean kinetic energy of translationally invariant states.

Theorem 5. The mean kinetic energy functional $T$, defined over the set $E \cap L_{R^{v}}^{\perp}$ of translationally invariant states over $\mathfrak{A}$, by

$$
T(\varrho)=\lim _{l_{1} \ldots l_{v} \rightarrow \infty} T_{\Lambda_{l}}(\varrho) / V\left(\Lambda_{l}\right)
$$

exists and has the following properties

0. $T(\varrho) \in[0,+\infty]$.

1. $T$ is affine.

2. $T$ is lower semi-continuous.

3. If $\varrho \in E \cap L_{R^{v}}^{\perp}$ and $\mu_{\varrho}$ is the unique probability measure with barycentre $\varrho$ concentrated on $\mathscr{E}\left(E \cap L_{R^{v}}^{\perp}\right)$ then

$$
T(\varrho)=\int d \mu_{\varrho}\left(\varrho^{\prime}\right) T\left(\varrho^{\prime}\right) .
$$

4. If the domain $F_{T}$ of $T$ is defined by

$$
F_{T}=\left\{\varrho ; \varrho \in E \cap L_{R^{v}}^{\perp}, T(\varrho)<+\infty\right\}
$$

then $\varrho \in F_{T}\left(\in F_{N} \cap F_{T}\right)$ implies that $\mu_{\varrho}$ is concentrated on $\mathscr{E}\left(E \cap L_{R^{\nu}}^{\perp}\right) \cap F_{T}$ $\left(\mathscr{E}\left(E \cap L_{R^{v}}^{\perp}\right) \cap F_{N} \cap F_{T}\right)$.

5. If $\varrho_{1}, \varrho_{2} \in \mathscr{E}\left(E \cap L_{R \nu}^{\perp}\right) \cap F_{T}$ and $T\left(\varrho_{1}\right) \neq T\left(\varrho_{2}\right)$ then $\varrho_{1}$ and $\varrho_{2}$ generate quasi-inequivalent representations. 
6. The set $F_{N} \cap F_{T}$ is weakly dense among the set of locally normal translationally invariant states and $\varrho \in F_{N} \cap F_{T}$ implies that

a) $T(\varrho)=T_{\Lambda}(\varrho) / V(\Lambda)$ for all $\Lambda \subset R^{v}$,

b) $T(\varrho) \geqq \frac{1}{2} \pi^{2} v\left(\frac{2 N(\varrho)}{v+2}\right)^{\frac{v+2}{v}}$.

Proof. The existence of $T$ follows in a standard manner from the increase and super additivity properties derived in Lemma 2 and indeed one has

$$
T(\varrho)=\sup _{l_{1} \ldots l_{\nu}} T_{\Lambda_{l}}(\varrho) / V\left(\Lambda_{l}\right)
$$

Thus $T$ is the supremum of a family of lower semi-continuous functions and Property 2 is evident. Properties 0 and 1 follow immediately; 3 is a consequence of 1 and $2 ; 4$ and 5 are proved in the same manner as similar properties given in Theorem $2 ; 6 a$ follows as $T_{A}(\varrho)$ is an additive function of $\Lambda$ if $\varrho \in F_{N} \cap F_{T}$ and $6 b$ is proved as follows. For $\varrho \in F_{N} \cap F_{T}$

$$
\begin{aligned}
T(\varrho) & =T_{\Lambda_{l}}(\varrho) / V\left(\Lambda_{l}\right)=\operatorname{Tr}_{\mathscr{H}_{F}\left(\Lambda_{l}\right)}\left(\varrho_{\Lambda_{l}} T_{\Lambda_{l}}\right) \\
& =\sum_{n} \varepsilon(n) \operatorname{Tr}_{\mathscr{H}_{F}\left(\Lambda_{l}\right)}\left(\varrho_{\Lambda_{l}} \pi_{\Lambda_{l}}\left(a\left(C_{\Lambda_{l}}^{n}\right) * a\left(C_{\Lambda_{l}}^{n}\right)\right) / V\left(\Lambda_{l}\right)\right. \\
& \geqq\left(\frac{\pi}{l_{1}}\right)^{2}\left[N(\varrho)-\frac{1}{l_{1} l_{2} \ldots l_{v}}\right]
\end{aligned}
$$

for $l_{1} \geqq l_{2}, \ldots l_{v}$ (we use the notation introduced in the discussion before Definition 2). Choosing $l_{1}=l_{2}=\cdots=l_{v}=(2 N(\varrho) / v+2)^{1 / v}$ we find the maximum of the right hand side gives the lower bound quoted. It remains to prove the density property of $F_{N} \cap F_{T}$.

Let $\varrho_{1}$ be an arbitrary invariant locally normal state and consider the weak neighbourhoods

$$
\mathscr{V}_{\varrho_{1}, A, \varepsilon}=\left\{\varrho ; \varrho \in E \cap L_{R v}^{\perp},\left|\varrho(A)-\varrho_{1}(A)\right|<\varepsilon\right\}
$$

Take $A_{1} \in \mathfrak{A}\left(\Lambda_{1}\right)$ such that $\left\|A-A_{1}\right\|<\varepsilon / 6$ and let $\Lambda$ be a parallelepiped such that $\Lambda_{1} \subset \Lambda$ and $\left(V\left(\Lambda_{2}\right) / V(\Lambda)\right)\left\|A_{1}\right\|<\varepsilon / 6$ where

$$
\Lambda_{2}=\bigcup_{x \in R^{v}}^{\prime}\left(\Lambda_{1}+x\right)
$$

and the prime indicates the restrictions $\Lambda_{1}+x \cap \Lambda \neq \theta$ and $\Lambda_{1}+x \nsubseteq \Lambda$ are to be placed on the union. Consider the Fock space $\mathscr{H}_{F}(\Lambda)$ and a basis of it constructed with functions $f_{\Lambda} \in \boldsymbol{C}_{0}^{\infty}(\Lambda)$ and their antisymmetrized products. Denote by $E_{m}$ the projector on a subspace of $\mathscr{H}_{F}(\Lambda)$ spanned by a finite number $m$ of elements of this basis. For each vector $\Psi \in E_{m} \mathscr{H}_{F}(\Lambda)$ the sum of the kinetic energies of the particles is bounded. Now $\varrho_{1} \mid \mathfrak{A}(\Lambda)$ is determined by a density matrix $\varrho_{1 \Lambda}$ and we now define $\varrho_{\Lambda}^{\prime}=E_{m} \varrho_{1 \Lambda} E_{m}$. 
Using $\varrho_{A}^{\prime}$ we construct a periodic state $\varrho^{\prime}$ over $\mathfrak{U}$ in a standard manner; in each parallelepiped $\Gamma \subset R^{v}$ which is formed by a finite union of disjoint translates of $\Lambda$ we define $\varrho_{\Gamma}^{\prime \prime}$ to be the tensor product of the $\varrho_{A}^{\prime}$ and we define the density matrix corresponding to any other bounded sèt in $R^{v}$ by use of the compatibility conditions. Finally we construct an invariant state $\varrho^{\prime \prime}$ by averaging $\varrho^{\prime}$ over translations i.e.

$$
\varrho^{\prime \prime}(B)=\frac{1}{V(\Lambda)} \int_{\Lambda} d x \varrho^{\prime}\left(\tau_{x} B\right), \quad B \in \mathfrak{U} .
$$

By construction it follows that $\varrho^{\prime \prime} \in F_{T} \cap F_{N}$ but we now show that the neighbourhood $\mathscr{V}_{Q_{1}, A, \varepsilon}$ contains such states. Note that

$$
\begin{aligned}
\left|\varrho^{\prime \prime}(A)-\varrho_{1}(A)\right| & \leqq 2\left\|A-A_{1}\right\|+\left|\varrho^{\prime \prime}\left(A_{1}\right)-\varrho_{1}\left(A_{1}\right)\right| \\
& <\frac{\varepsilon}{3}+\left|\varrho^{\prime \prime}\left(A_{1}\right)-\varrho_{1}\left(A_{1}\right)\right|
\end{aligned}
$$

and

Thus we have

$$
\left|\varrho^{\prime \prime}\left(A_{1}\right)-\operatorname{Tr}_{\mathscr{H}_{F}(\Lambda)}\left(\varrho_{\Lambda}^{\prime} \pi_{\Lambda}\left(A_{1}\right)\right)\right| \leqq 2 V\left(\Lambda_{2}\right) / V(\Lambda)\left\|A_{1}\right\| \leqq \frac{\varepsilon}{3} .
$$

$$
\left|\varrho^{\prime \prime}(A)-\varrho_{1}(A)\right|<\frac{2}{3} \varepsilon+\left|\operatorname{Tr}_{\mathscr{H}_{F}(\Lambda)}\left(\left(\varrho_{\Lambda}^{\prime}-\varrho_{1 \Lambda}\right) \pi_{\Lambda}\left(A_{1}\right)\right)\right| .
$$

The right hand side can however be made less than $\varepsilon$ by choosing $m$ sufficiently large. This proves that all weak neighbourhoods of locally normal states contain states belonging to $F_{N} \cap F_{T}$.

Finally we note that the properties of the locally normal invariant states with finite mean kinetic energy, i.e. the states $\varrho \in F_{N} \cap F_{T}$, can be expressed in terms of unbounded operators.

Theorem 6. Let $\varrho$ be a locally normal invariant state with finite mean kinetic energy $T(\varrho)$ and let $\left(\pi_{\varrho}, U_{\varrho}, \mathscr{H}_{\varrho}, \Omega_{\varrho}\right)$ be the representation of $\mathfrak{A}$, the unitary representation of $R^{\prime}$, the Hilbert space, and cyclic invariant vector, associated with $\varrho$ by the Gelfand-Segal construction.

It follows that the mean density $N(\varrho)$ of $\varrho$ is finite and hence the conclusions of Theorem 3 are valid. Further it follows that there exists a positive self-adjoint operator $T_{\varrho}$ on $\mathscr{H}_{\varrho}$ with the following properties.

1. $T(\varrho)=\left(\Omega_{\varrho}, T_{\varrho} \Omega_{\varrho}\right)$ and in particular $\Omega_{\varrho} \in \mathscr{D}\left(T_{\varrho}^{\frac{1}{2}}\right)$, the domain of $T_{\varrho}^{\frac{1}{2}}$.

2. The following commutation relations are valid

$$
\begin{array}{ll}
{\left[T_{\varrho}, \pi_{\varrho}(A)\right]=0,} & A \in \mathfrak{A}, \\
{\left[T_{\varrho}, U_{\varrho}(x)\right]=0,} & x \in R^{v},
\end{array}
$$

and in particular $\pi_{\varrho}$ and $U_{\varrho}$ leave the domain of $T_{\varrho}$ invariant. Hence $T_{\varrho}$ is affiliated with the abelian algebra $\left\{\pi_{\varrho}, U_{\varrho}\right\}^{\prime}$ of operators on $\mathscr{H}_{\varrho}$. 
3. If $\varrho \in \mathscr{E}\left(E \cap L_{R v}^{+}\right) \cap F_{N} \cap F_{T}$ then $T_{\varrho}=T(\varrho) 1_{\varrho}$ where $1_{\varrho}$ is the identity operator on $\mathscr{H}_{\varrho}$.

Proof. That $N(\varrho)<+\infty$ is a consequence of property 0 of Theorem 5 . Now from Theorems 4 and 5 and the discussion of the Fock representation we have

$$
\begin{aligned}
T(\varrho) & =\frac{1}{V(\Lambda)} \operatorname{Tr}_{\mathscr{H}_{F}(\Lambda)}\left(\varrho_{\Lambda} T_{\Lambda}\right) \\
& =\frac{1}{V(\Lambda)} \sum_{n} \varepsilon(n) \operatorname{Tr}_{\mathscr{H}_{F}(\Lambda)}\left(\varrho_{\Lambda} \pi_{\Lambda}\left(a\left(C_{\Lambda}^{n}\right)^{*} a\left(C_{\Lambda}^{n}\right)\right)\right) \\
& =\frac{1}{V(\Lambda)} \sum_{n} \varepsilon(n) \varrho\left(a\left(C_{\Lambda}^{n}\right)^{*} a\left(C_{\Lambda}^{n}\right)\right)
\end{aligned}
$$

for all parallelepipeds $\Lambda$. This last form of $T(\varrho)$ allows us to repeat the arguments used in establishing Theorem 3 . One first deduces that for a dense set of $A \in \mathfrak{A}$, namely polynomials in $a\left(C_{A}^{n}\right)^{*}, a\left(C_{A}^{m}\right)$ the limit

$$
T\left(\varrho_{A}\right)=\operatorname{Lim}_{l_{1} \ldots l_{\nu} \rightarrow \infty} \frac{1}{V(\Lambda)} \sum_{n} \varepsilon(n) \varrho\left(A^{*} a\left(C_{A}^{n}\right)^{*} a\left(C_{\Lambda}^{n}\right) A\right)
$$

exists and then one constructs an operator $T_{\varrho}$ such that

$$
\begin{aligned}
& T\left(\varrho_{A}\right)=\left(\Omega_{\varrho} \pi_{\varrho}\left(A^{*}\right) T_{\varrho} \pi_{\varrho}(A) \Omega_{\varrho}\right) \\
& T\left(\varrho_{A}\right)=\left(\Omega_{\varrho} \pi_{\varrho}\left(A^{*}\right) T_{\varrho} \pi_{\varrho}(A) \Omega_{\varrho}\right)
\end{aligned}
$$

(note that as $\varrho$ is locally normal $T_{A}\left(\varrho_{A}\right)$ is additive by Lemma 2). As the proof is essentially identical to that of Theorem 3 we will not repeat the details.

\section{Entropy and Mean Entropy}

The aim of this section is to give a definition of the mean entropy of translationally invariant states over $\mathfrak{A}$. As we shall see however this problem is rather difficult in general and we only obtain a satisfactory solution by restricting discussion to the set of locally normal states with finite mean kinetic energy. The difficulties which arise stem from states which describe physical configurations with such a high degree of disorder that the entropy is infinite. Heuristically one would expect large disorder to be associated with high kinetic energy and conversely one might expect states with finite mean kinetic energy to have finite mean entropy. We will demonstrate that this latter expectation is indeed justified by analysing the entropy with the mean kinetic energy fixed. 
Before proceeding let us make a few heuristic comments on the connection between the definition of the entropy and our foregoing discussion of the density and kinetic energy. If $\varrho$ is a normal state over $\mathfrak{U}(\Lambda)$ determined by the density matrix $\varrho_{A}$ then the local entropy is naturally introduced as a functional $S_{A}$ over $E_{\mathscr{U}(\Lambda)}$ by

$$
S_{\Lambda}(\varrho)=-\operatorname{Tr}_{\mathscr{H}_{F}(\Lambda)}\left(\varrho_{\Lambda} \log \varrho_{\Lambda}\right) .
$$

However we can also rewrite this definition on $\mathscr{H}_{Q}$ the carrier space of the representation $\pi_{\varrho}$ generated by $\varrho$. Noting that $\varrho_{A} \in \pi_{A}^{\prime \prime}$ we can identify $\varrho_{A}$, as an operator on $\mathscr{H}_{\varrho}$. with an element $B_{\varrho} \in \pi_{\varrho}^{\prime \prime}$. Hence

$$
S_{\Lambda}(\varrho)=-\left(\Omega_{\varrho}, \log B_{\varrho} \Omega_{\varrho}\right)
$$

is the extension of the state $\varrho$ to an unbounded operator $-\log B_{\varrho}$ which is affiliated with $\pi_{\varrho}^{\prime \prime}$. [Note that $\log B_{\varrho}$ only has a dense domain of definition if $\varrho_{\Lambda}$ is invertible e.g. if $\Omega_{\varrho}$ is separating for $\pi_{\varrho}$.] This is the same situation encountered with the number operator etc. but now the unbounded operator occurring has a direct connection with the state $\varrho$ rather than the representation $\pi_{e}$. As a consequence we have to adopt different techniques of analysis.

The methods which we will use were first developed in [10] but we will need strengthened versions of the results given in this reference. The essential statements can be phrased as theorems concerning states over algebras of compact operators. As this is possibly of interest in its own right we develop the results in the following separate subsection.

\section{a) Algebra of Compact Operators}

Let $\mathscr{H}$ be a Hilbert space and consider the $C^{*}$-algebra $\mathscr{L} \mathscr{C}(\mathscr{H})$ of compact operators on $\mathscr{H}$. Recall that each state $\varrho$ over $\mathscr{L} \mathscr{C}(\mathscr{H})$ is determined by a density matrix $\hat{\varrho}$ on $\mathscr{H}$, i.e. each state is normal with respect to the basic defining representation of the algebra. Thus we can define the entropy $S$ as a function over the states of $\mathscr{L} \mathscr{C}(\mathscr{H})$ as follows

$$
\begin{aligned}
S(\varrho) & =-\operatorname{Tr}_{\mathscr{H}}(\hat{\varrho} \log \hat{\varrho}) & & \text { if } \hat{\varrho} \log \hat{\varrho} \text { is of trace class } \\
& =+\infty & & \text { otherwise. }
\end{aligned}
$$

We will also have cause to consider a related concept, the relative, or conditional, entropy. Let $\sigma$ be a fixed state over $\mathscr{L} \mathscr{C}(\mathscr{H})$; the relative entropy $S_{\sigma}$ is defined as a function over the states of $\mathscr{L} \mathscr{C}(\mathscr{H})$ by

$$
\begin{aligned}
S_{\sigma}(\varrho) & =-\operatorname{Tr}_{\mathscr{H}}(\hat{\varrho} \log \hat{\varrho}-\hat{\varrho} \log \hat{\sigma}) \quad \text { if } \hat{\varrho} \log \hat{\varrho}-\hat{\varrho} \log \hat{\sigma} \text { is of trace class } \\
& =-\infty \text { otherwise. }
\end{aligned}
$$


The analysis of the properties of $S$ and $S_{\sigma}$ is aided by the following result

Lemma 4. Let $\varrho$ and $\sigma$ be positive trace class operators on $\mathscr{H}$ then if $\Psi \in \mathscr{H}$ is an eigenvector of $\varrho$, or of $\sigma$, the following inequality is valid

$$
-(\Psi,\{\varrho \log \varrho-\varrho \log \sigma-\varrho+\sigma\} \Psi) \leqq 0
$$

if, further, $\sigma \geqq \varrho$ then

$$
0 \leqq-(\Psi,\{\varrho \log \varrho-\varrho \log \sigma\} \Psi)
$$

for each eigenvector $\Psi$ of $\varrho$.

Proof. This result is contained, but not stated, in [10]. The first statement follows by using the convexity of the function $x \rightarrow-x \log x$ in the form

$$
-x(\log x-\log y)+x-y \leqq 0, \quad x, y \geqq 0 .
$$

Further the convexity of the function $x \rightarrow \log x$ implies that

$$
(\Psi, \log \sigma \Psi) \leqq \log (\Psi, \sigma \Psi)
$$

for each normalized $\Psi \in \mathscr{H}$, whilst the convexity of $x \rightarrow-x \log x$ implies that

$$
-(\Psi, \varrho \log \varrho \Psi) \leqq-(\Psi, \varrho \Psi) \log (\Psi \varrho \Psi) .
$$

The first statement of the lemma follows by a straightforward combination of these inequalities.

The second statement is a consequence of the fact that $\sigma \geqq \varrho$ implies that

$$
\log \sigma \geqq \log \varrho
$$

a property which follows from the theory of monotone operator functions.

Application of the above lemma now leads to the following result concerning the entropy $S$ and the conditional entropy $S_{\sigma}$.

Lemma 5. The entropy $S$ as a function over the states of $\mathscr{L} \mathscr{C}(\mathscr{H})$ satisfies the extremal principles

$$
\begin{aligned}
S(\varrho) & =\inf _{\tau}-\operatorname{Tr}_{\mathscr{H}}(\hat{\varrho} \log \tau-\tau)-1 \\
& =\sup _{\tau}-\operatorname{Tr}_{\mathscr{H}}(\varrho \log (\varrho+\tau))
\end{aligned}
$$

where the sup and inf are taken over the class of positive trace class operators $\tau$ on $\mathscr{H}$. Hence $S$ is positive and such that

1. The following inequalities are valid for $0 \leqq \lambda \leqq 1$

a) $S\left(\lambda \varrho_{1}+(1-\lambda) \varrho_{2}\right) \geqq \lambda S\left(\varrho_{1}\right)+(1-\lambda) S\left(\varrho_{2}\right)$

b) $S\left(\lambda \varrho_{1}+(1-\lambda) \varrho_{2}\right) \leqq \lambda S\left(\varrho_{1}\right)+(1-\lambda) S\left(\varrho_{2}\right)-\lambda \log \lambda-(1-\lambda) \log (1-\lambda)$ 
2. $S$ is lower semi-continuous in the weak topology.

The conditional entropy $S_{\sigma}$ has the properties

0. $S_{\sigma}(\varrho) \in[0,-\infty]$.

1. The inequalities $1 a$ and $1 b$ above are valid with the replacement of $S$ by $S_{\sigma}$.

2. $S_{\sigma}$ is upper semi-continuous in the weak* topology.

Proof. From the foregoing lemma we have

$$
\begin{aligned}
-\operatorname{Tr}_{\mathscr{H}}(\varrho \log (\varrho+\tau)) & \leqq-\operatorname{Tr}_{\mathscr{H}}(\hat{\varrho} \log \hat{\varrho}) \\
& \leqq-\operatorname{Tr}_{\mathscr{H}}(\varrho \log \tau+\hat{\varrho}-\tau)
\end{aligned}
$$

for $\tau \geqq 0$ of trace class and hence the extremal principles follow. From the first extremal principle we have

$$
\begin{aligned}
S\left(\lambda \varrho_{1}+(1-\lambda) \varrho_{2}\right)= & \inf _{\tau}\left\{-\lambda \operatorname{Tr}_{\mathscr{H}}\left(\varrho_{1} \log \tau-\tau\right)-\lambda\right. \\
& \left.-(1-\lambda) \operatorname{Tr}_{\mathscr{H}}\left(\varrho_{2} \log \tau-\tau\right)-(1-\lambda)\right\} \\
\geqq & \lambda \inf _{\tau}\left\{-\operatorname{Tr}_{\mathscr{H}}\left(\varrho_{1} \log \tau-\tau\right)-1\right\} \\
& +(1-\lambda) \inf _{\tau}\left\{-\operatorname{Tr}_{\mathscr{H}}\left(\hat{\varrho}_{2} \log \tau-\tau\right)-1\right\} \\
= & \lambda S\left(\varrho_{1}\right)+(1-\lambda) S\left(\varrho_{2}\right) .
\end{aligned}
$$

The second inequality follows from a similar calculation using the second extremal principle; we omit the details.

To prove the lower semi-continuity of $S$ we use the second variational principle in the slightly different form

$$
S(\varrho)=\sup _{1 \geqq \tau \geqq \hat{\varrho}}-\operatorname{Tr}_{\mathscr{H}}(\varrho \log \tau) .
$$

Now let $\left(\Psi_{\varrho}^{i}\right)_{i \geqq 1}$ and $\left(\Psi_{\tau}^{j}\right)_{j \geqq 1}$ be complete orthonormal basis in $\mathscr{H}$ formed by eigenvectors of $\varrho$ and $\tau$ respectively. Then using $\varrho \geqq 0$ and $\log \tau \leqq 0$ we have

$$
S(\varrho)=\sup _{1 \geqq \tau \geqq \varrho \hat{N}, M} \sup _{N, M}-\sum_{\substack{i \leq N \\ j \geqq N}}\left(\Psi_{\varrho}^{i}, \hat{\varrho} \Psi_{\varrho}^{i}\right)\left|\left(\Psi_{\varrho}^{i}, \Psi_{\tau}^{j}\right)\right|^{2} \log \left(\Psi_{\tau}^{i}, \tau \Psi_{\tau}^{j}\right) .
$$

It follows however from perturbation theory that the expression occurring on the right hand side is continuous in the weak* topology on $E_{\mathscr{L} \mathscr{C}(\mathscr{H})}$. Hence $S$ is the upper envelope of a family of continuous functions i.e. $S$ is lower semi-continuous.

The negativity of $S_{\sigma}$ follows from its definition and the first statement of Lemma 4 . As $S_{\sigma}$ and $S$ differ by an affine function only it follows that $S_{\sigma}$ satisfies extremal principles similar to those given for $S$ and hence 
similar inequalities are valid. If $\left(\Psi_{\varrho}^{i}\right)_{i \geqq 1}$ and $\left(\Psi_{\sigma}^{j}\right)_{j \geqq 1}$ are now complete orthonormal basis in $\mathscr{H}$ spanned by eigenvectors of $\hat{\varrho}$ and $\hat{\sigma}$ respectively we then have as a consequence of the above definitions and the first statement of Lemma 4 that

$$
\begin{aligned}
S_{\sigma}(\varrho)= & \inf _{N M}\left[-\sum_{i \leqq N}\left(\Psi_{\varrho}^{i},\{\hat{\varrho} \log \hat{\varrho}-\hat{\varrho}+\hat{\sigma}\} \Psi_{\varrho}^{i}\right)\right. \\
& \left.+\sum_{\substack{i \leqq N \\
j \leqq M}}\left(\Psi_{\varrho}^{i} \hat{\varrho} \Psi_{\varrho}^{i}\right)\left|\left(\Psi_{\varrho}^{i}, \Psi_{\sigma}^{j}\right)\right|^{2} \log \left(\Psi_{\sigma}^{j} \hat{\sigma} \Psi_{\sigma}^{j}\right)\right]
\end{aligned}
$$

But, again from perturbation theory, the function occurring on the right hand side is continuous in the weak* topology and hence $S_{\sigma}$ is upper semi-continuous.

We now apply the above information to the states of the Fermi system.

\section{b) Algebra of Fermions}

We begin by considering the local entropy and the local conditional entropy. To introduce this latter concept we use the kinetic energy which we have previously discussed. The principal definition is the following.

Definition 3. The local entropy $S_{A}$ is defined as a functional over the states of $\mathfrak{A}(\Lambda)$ which are normal with respect to the Fock representation by

$$
S_{\Lambda}(\varrho)=-\operatorname{Tr}_{\mathscr{H}_{F}(\Lambda)}\left(\varrho_{A} \log \varrho_{A}\right) .
$$

If the density matrix $\varrho_{A}$ which determines $\varrho$ is such that $\varrho_{\Lambda} \log \varrho_{A}$ is of trace class; if this latter condition is not satisfied then we define $S_{A}(\varrho)=+\infty$.

For each $\beta>0$ a conditional entropy is defined over the normal states of $\mathfrak{A}(\Lambda)$ by

$$
S_{\Lambda, \beta}(\varrho)=-\operatorname{Tr}_{\mathscr{H}_{F}(\Lambda)}\left(\varrho_{A} \log \varrho_{\Lambda}-\beta \varrho_{A} T_{A}\right)
$$

if the operator occurring on the right hand side is of trace class and by $S_{A, \beta}(\varrho)=-\infty$ if this is not the case $\left[T_{A}\right.$ is the kinetic energy operator on Fock space].

Two remarks are in order concerning this definition. Firstly we could extend the definition of $S_{\Lambda}$ and $S_{\Lambda, \beta}$ to all states over $\mathfrak{A}(\Lambda)$ by assigning the values $S_{\Lambda}(\varrho)=+\infty, S_{\Lambda, \beta}(\varrho)=-\infty$ if $\varrho$ is not normal with respect to the Fock representation. We have refrained from doing this because of our inability to discuss the continuity properties of $S_{A}$ and $S_{A, \beta}$ over all states in $E_{\mathscr{Q}(\Lambda)}$ (see below). Secondly we could have introduced a conditional entropy by first defining a density matrix $\sigma_{\Lambda, \beta}$ on $\mathscr{H}_{F}(\Lambda)$ by

$$
\sigma_{\Lambda, \beta}=e^{-\beta T_{\Lambda} / \operatorname{Tr}_{\mathscr{H}_{F}(\Lambda)}\left(e^{-\beta T_{\Lambda}}\right)}
$$


and then considering

$$
\begin{aligned}
\hat{S}_{\Lambda, \beta}(\varrho) & =-\operatorname{Tr}_{\mathscr{H}_{F}(\Lambda)}\left(\varrho_{\Lambda} \log \varrho_{\Lambda}-\varrho_{\Lambda} \log \sigma_{\Lambda, \beta}\right) \\
& =S_{\Lambda, \beta}(\varrho)-\log \operatorname{Tr}_{\mathscr{H}_{F}(\Lambda)}\left(e^{-\beta T_{\Lambda}}\right) .
\end{aligned}
$$

Mathematically $\hat{S}_{\Lambda, \beta}$ is a more natural object to consider than $S_{A, \beta}$; however physically the latter functional is of the most direct relevance. As $\hat{S}_{A, \beta}$ and $S_{A, \beta}$ only differ by a constant the properties of the former are easily derivable from the latter.

The results of the previous subsection can now be applied to obtain the following properties of $S_{A}$ and $S_{A, \beta}$.

Theorem 7. Let $K \subset E_{\mathscr{U}(1)}$ be any convex weakly compact subset of locally normal states. The function $\varrho \in K \rightarrow S(\varrho)$ has the following properties:

0. $S_{\Lambda}(\varrho) \in[0,+\infty]$.

1. $S_{A}$ is lower semi-continuous over $K$.

2. If $\varrho_{1}, \varrho_{2} \in K$ and $0 \leqq \lambda \leqq 1$ then the following inequalities are valid.

$S_{\Lambda}\left(\lambda \varrho_{1}+(1-\lambda) \varrho_{2}\right) \geqq \lambda S_{\Lambda}\left(\varrho_{1}\right)+(1-\lambda) S_{\Lambda}\left(\varrho_{2}\right)$,

$S_{\Lambda}\left(\lambda \varrho_{1}+(1-\lambda) \varrho_{2}\right) \leqq \lambda S_{\Lambda}\left(\varrho_{1}\right)+(1-\lambda) S_{\Lambda}\left(\varrho_{2}\right)-\lambda \log \lambda-(1-\lambda) \log (1-\lambda)$.

The function $\varrho \in K \rightarrow S_{\Lambda, \beta}(\varrho)$ has the properties

0. $S_{\Lambda, \beta}(\varrho) \in\left[-\infty, \log \operatorname{Tr}_{\mathscr{H}_{F}(\Lambda)}\left(e^{-\beta T_{A}}\right)\right]$.

1. $S_{A, \beta}$ is upper semi-continuous over $K$.

2. The inequalities 2 above are valid with $S_{\Lambda}$ replaced by $S_{A, \beta}$.

The following conditions are equivalent:

1. $T_{\Lambda}(\varrho)<+\infty$ and $N_{\Lambda}(\varrho)<+\infty$.

2. $S_{\Lambda, \beta}(\varrho)>-\infty$ for one $\beta>0$.

3. $S_{\Lambda, \beta}(\varrho)>-\infty$ for all $\beta>0$

and each of these conditions implies that

$$
0 \leqq S_{\Lambda}(\varrho) \leqq \beta T_{\Lambda}(\varrho)+\log \operatorname{Tr}_{\mathscr{H}_{F}(\Lambda)}\left(e^{-\beta T_{\Lambda}}\right)<+\infty .
$$

Proof. The properties of $S_{A}$ and $S_{A, \beta}$ are similar to those obtained in Lemma 5 for the corresponding quantities discussed in the previous subsection and the proofs are identical. In the present case one uses the assumed weak compactness of $K$ to discuss the continuity properties. We will not discuss the details of the derivation of these properties further.

To prove the equivalence of the three conditions we note that from the definition of $S_{A, \beta}$ and Condition 1 we have

$$
-\infty<-\beta T_{\Lambda}(\varrho) \leqq S_{\Lambda, \beta}(\varrho)
$$


and hence 3 is satisfied. However Condition 3 trivially implies Condition 2 and it remains to prove that 2 implies 1 . Take $0<\beta_{0}<\beta$ then using the upper bound for $S_{\Lambda, \beta-\beta_{0}}$ we have that

$$
\begin{aligned}
0 & \leqq \beta_{0} \operatorname{Tr}_{\mathscr{H}_{F}(A)}\left(\varrho_{A} T_{A}\right) \\
& \leqq-S_{A, \beta}(\varrho)+\log \operatorname{Tr}_{\mathscr{H}_{F}(\Lambda)}\left(e^{-\left(\beta-\beta_{0}\right) T_{A}}\right) .
\end{aligned}
$$

Hence $\varrho_{A} T_{A}$ is of trace class and Condition 1 follows from Theorem 4. The upper bound on $S_{A}$ follows directly from the upper bound on $S_{A, \beta}$.

Note that it is possible that $S_{\Lambda}(\varrho)<+\infty$ but $T_{\Lambda}(\varrho)=+\infty$ as can be seen from explicit examples.

Next we wish to examine the properties of the functions $\Lambda \rightarrow S_{\Lambda}(\varrho)$ and $\Lambda \rightarrow S_{\Lambda, \beta}(\varrho)$ with $\varrho$ fixed. Actually the necessary results have already been obtained in [10] and we recall them in the following lemma.

Lemma 6. Let $\Lambda$ and $\Lambda^{\prime}$ be disjoint bounded open sets in $R^{v}$ and let $\varrho$ be an even state over $\mathfrak{A}\left(\Lambda \cup \Lambda^{\prime}\right)$. The entropy $S_{\Lambda}(\varrho)$ and conditional entropy $S_{\Lambda, \beta}(\varrho)$ then satisfy the subadditivity properties

$$
\begin{aligned}
S_{\Lambda \cup A^{\prime}}(\varrho) & \leqq S_{\Lambda}(\varrho)+S_{\Lambda^{\prime}}(\varrho), \\
S_{A \cup \Lambda^{\prime}, \beta}(\varrho) & \leqq S_{\Lambda, \beta}(\varrho)+S_{\Lambda^{\prime}, \beta}(\varrho), \quad \Lambda \cap \Lambda^{\prime}=\theta .
\end{aligned}
$$

If $\varrho \in E \cap L_{R v}^{\perp}$ is a translationally invariant state over $\mathfrak{A}$ then

$$
S_{A+a}(\varrho)=S_{\Lambda}(\varrho), S_{A+a, \beta}(\varrho)=S_{\Lambda, \beta}(\varrho), \quad a \in R^{v} .
$$

We refer to [10] for the proof of the lemma; again it is dependent upon the inequalities of Lemma 4. [Actually only properties of $S_{A}(\varrho)$ are derived in [10] but the properties of $S_{A, \beta}(\varrho)$ then follow directly from Definition 3 and Lemma 3.]

Our next aim is to introduce an entropy per unit volume for suitable translationally invariant states over $\mathfrak{A}$. It is well known that a mean value of this type can be defined if $\Lambda \rightarrow S_{\Lambda}(\varrho)$ is a sub-additive, invariant, set function with certain measurability properties. It is in the last point that the functions $\Lambda \rightarrow S_{\Lambda}(\varrho)$ and $\Lambda \rightarrow S_{\Lambda, \beta}(\varrho)$ differ. We first consider the mean conditional entropy as a basis for a later discussion of the mean entropy.

Theorem 8. Let $\Lambda \subset R^{v}$ be a bounded open parallelepiped with edges of length $l_{1}, l_{2}, \ldots l_{v}$, and let @ be a translationally invariant locally normal state over $\mathfrak{A}$. The following limit exists

$$
S_{\beta}(\varrho)=\operatorname{Lim}_{l_{1} \ldots l_{v} \rightarrow \infty} \frac{S_{\Lambda, \beta}(\varrho)}{V(\Lambda)}, \quad \beta>0
$$


and defines a mean conditional entropy with the following properties

o. $S_{\beta}(\varrho) \in\left[-\infty, \frac{1}{(4 \pi \beta)^{v / 2}}\right]$

1. $S_{\beta}(\varrho)=\inf _{l_{1}, \ldots l_{v}} \frac{S_{\Lambda, \beta}(\varrho)}{V(\Lambda)}$.

2. $S_{\beta}$ is affine i.e. if $\varrho_{1}, \varrho_{2} \in E \cap L_{R^{v}}^{\perp}$ are both locally normal and $0 \leqq \lambda \leqq 1$ then

$$
S_{\beta}\left(\lambda \varrho_{1}+(1-\lambda) \varrho_{2}\right)=\lambda S_{\beta}(\varrho)+(1-\lambda) S_{\beta}\left(\varrho_{2}\right) .
$$

3. Let $K \subset E \cap L_{R^{v}}^{\perp}$ be any weakly compact set of locally normal states. $S_{\beta}$ is upper semi-continuous over $K$.

4. If $\varrho \in E \cap L_{R^{v}}^{\perp}$ is locally normal and $\mu_{\varrho}$ is the unique measure with barycentre $\varrho$ concentrated on $\mathscr{E}\left(E \cap L_{R^{v}}^{\perp}\right)$ then

$$
S_{\beta}(\varrho)=\int d \mu_{\varrho}\left(\varrho^{\prime}\right) S_{\beta}\left(\varrho^{\prime}\right)
$$

Proof. From Theorem 7 we have

$$
S_{\Lambda, \beta}(\varrho) \leqq \log \operatorname{Tr}_{\mathscr{H}_{F}(\Lambda)}\left(e^{\left.-\beta T_{\Lambda}\right)}\right)
$$

and using the estimate of the trace given in the previous section

$$
S_{\Lambda, \beta}(\varrho) \leqq V(\Lambda)\left(\frac{1}{(4 \pi \beta)^{v / 2}}\right) \prod_{i=1}^{v}\left(1+\frac{\sqrt{4 \pi \beta}}{l_{i}}\right)
$$

for each parallelepiped $\Lambda$ with sides of length $l_{1}, \ldots l_{v}$. This property together with the sub-additivity and invariance of the set function $\Lambda \rightarrow S_{\Lambda, \beta}(\varrho)$ ensures, by a standard argument, the existence of $S_{\beta}(\varrho)$ and the first two properties quoted. The affine property follows from Property 2 of Theorem 7 and the lower semi-continuity arises because $S_{\beta}$ is given as the infimum of the family $V(\Lambda)^{-1} S_{\beta, \Lambda}$ of upper semi-continuous functions. It remains to prove Property 4.

Let $K$ be a convex weakly compact set of locally normal states and decompose $\varrho$ in the form

where

$$
\varrho=\varrho_{K} \mu_{\varrho}(K)+\varrho_{R}\left(1-\mu_{\varrho}(K)\right)
$$

$$
\varrho_{K}=\frac{1}{\mu_{\varrho}(K)} \int_{K} d \mu_{\varrho}\left(\varrho^{\prime}\right) \varrho^{\prime} \text {. }
$$

As $\mu_{\varrho}$ can be weakly approximated by a finite sum of point measures we deduce that $\varrho_{K} \in K$. Now as $S_{\beta}$ is affine, and also upper semi-continuous over $K$, we have

$$
S_{\beta}(\varrho)=\int_{K} d \mu_{\varrho}\left(\varrho^{\prime}\right) S_{\beta}\left(\varrho^{\prime}\right)+\left(1-\mu_{\varrho}(K)\right) S_{\beta}\left(\varrho_{R}\right) .
$$


However

and hence

$$
\frac{1}{(4 \pi \beta)^{v / 2}} \geqq S_{\beta}\left(\varrho_{R}\right) \geqq-\beta T\left(\varrho_{R}\right)
$$

$$
\begin{aligned}
\left(1-\mu_{\varrho}(K)\right) \frac{1}{(4 \pi \beta)^{v / 2}} & \geqq S_{\beta}(\varrho)-\int_{K} d \mu_{\varrho}\left(\varrho^{\prime}\right) S_{\beta}\left(\varrho^{\prime}\right) \\
& \geqq-\beta\left(1-\mu_{\varrho}(K)\right) T\left(\varrho_{R}\right) \\
& =-\beta\left(T(\varrho)-\int_{K} d \mu_{\varrho}\left(\varrho^{\prime}\right) T\left(\varrho^{\prime}\right)\right)
\end{aligned}
$$

where the last equality follows from Theorem 5 . Thus from this estimate and the decomposition properties of $T$ we deduce that if $K_{\alpha}$ is any net of convex weakly compact sets of locally normal states with the property

then it follows that

$$
\operatorname{Lim}_{\alpha} \mu_{\varrho}\left(K_{\alpha}\right)=1
$$

$$
S_{\beta}(\varrho)=\operatorname{Lim}_{\alpha} \int_{K_{\alpha}} d \mu_{\varrho}\left(\varrho^{\prime}\right) S_{\beta}\left(\varrho^{\prime}\right)
$$

i.e. $S_{\beta}$ is $\mu_{\varrho}$-measurable and

$$
S_{\beta}(\varrho)=\int d \mu_{\varrho}\left(\varrho^{\prime}\right) S_{\beta}\left(\varrho^{\prime}\right)
$$

This completes the proof of the theorem.

Thus we have established that

$$
S_{\beta}(\varrho)=\operatorname{Lim}_{l_{1} \ldots l_{v} \rightarrow \infty}\left[\frac{S_{\Lambda}(\varrho)}{V(\Lambda)}-\beta \frac{T_{\Lambda}(\varrho)}{V(\Lambda)}\right]
$$

exists for each locally normal state. Hence if we next restrict our attention to the locally normal states with finite kinetic energy we deduce the existence of the mean entropy $S$ where

$$
S(\varrho)=\operatorname{Lim}_{l_{1} \ldots l_{\nu} \rightarrow \infty} \frac{S_{\Lambda}(\varrho)}{V(\Lambda)}=S_{\beta}(\varrho)+\beta T(\varrho) .
$$

Although $S$ has no apparent continuity properties we can straightforwardly deduce a decomposition property through use of our knowledge of $S_{\beta}$ and $T$.

Theorem 9. Let $\Lambda \subset R^{v}$ be a bounded open parallelepiped with edges of length $l_{1}, \ldots l_{v}$. The following limit exists

$$
S(\varrho)=\operatorname{Lim}_{l_{1} \ldots l v \rightarrow \infty} \frac{S_{\Lambda}(\varrho)}{V(\Lambda)}
$$

for each $\varrho \in F_{N} \cap F_{T}$ and defines a mean entropy with the following properties.

$$
\text { 0. } T(\varrho) \geqq 2 \pi v\left(\frac{2 S(\varrho)}{v+2}\right)^{\frac{v+2}{2}}, \quad \varrho \in F_{N} \cap F_{T} .
$$


1. $S$ is affine over $F_{N} \cap F_{T}$.

2. If $\varrho \in F_{N} \cap F_{T}$ and $\mu_{\varrho}$ is the unique measure with barycentre $\varrho$ concentrated on $\mathscr{E}\left(E \cap L_{R v}^{\perp}\right) \cap F_{N} \cap F_{T}$ then

$$
S(\varrho)=\int d \mu_{\varrho}\left(\varrho^{\prime}\right) S\left(\varrho^{\prime}\right) \text {. }
$$

3. If $\varrho_{1}, \varrho_{2} \in \mathscr{E}\left(E \cap L_{R v}^{\perp}\right) \cap F_{N} \cap F_{T}$ and $S\left(\varrho_{1}\right) \neq S\left(\varrho_{2}\right)$ then the representations $\pi_{\varrho_{1}}, \pi_{\varrho_{2}}$ generated by $\varrho_{1}$ and $\varrho_{2}$ respectively are quasi-inequivalent.

Proof. As mentioned the existence of $S$ and Properties 1 and 2 can be deduced from Theorems 5 and 8 . Actually one could deduce the existence of $S$ from the property

$$
\begin{aligned}
0 \leqq S_{\Lambda}(\varrho) & \leqq \beta T_{\Lambda}(\varrho)+\log \operatorname{Tr}_{\mathscr{H}_{F}(\Lambda)}\left(e^{-\beta T_{\Lambda}}\right) \\
& \leqq V(\Lambda)\left\{\beta T(\varrho)+\frac{1}{(4 \pi \beta)^{\nu / 2}} \prod_{i=1}^{\nu}\left(1+\frac{\sqrt{4 \pi \beta}}{l_{i}}\right)\right\}
\end{aligned}
$$

for $\varrho \in F_{N} \cap F_{T}$, and the sub-additivity and invariance derived in Lemma 6. One then finds

$$
S(\varrho)=\inf _{l_{1} \ldots l_{v}} \frac{S_{\Lambda}(\varrho)}{V(\Lambda)} \leqq \beta T(\varrho)+\frac{1}{(4 \pi \beta)^{v / 2}} .
$$

Minimizing the upper bound with respect to $\beta$ we find property 0 above. Finally we note that if $S\left(\varrho_{1}\right)<S\left(\varrho_{2}\right)$ we can choose $\Lambda$ such that

$$
-\operatorname{Tr}_{\mathscr{H}_{F}(\Lambda)}\left(\varrho_{1 \Lambda} \log \varrho_{1 \Lambda}\right)<-\operatorname{Tr}_{\mathscr{H}_{F}(\Lambda)}\left(\varrho_{2 A} \log \varrho_{2 A}\right) .
$$

But then from Lemma 4 we have

$$
-\operatorname{Tr}_{\mathscr{H}_{F}(\Lambda)}\left(\varrho_{2 \Lambda} \log \varrho_{2 \Lambda}\right)<-\operatorname{Tr}_{\mathscr{H}_{F}(\Lambda)}\left(\varrho_{2 \Lambda} \log \varrho_{1 \Lambda}\right) .
$$

However we can choose a sequence $A_{i} \in \mathfrak{A}(\Lambda)$ such that $\pi_{\Lambda}\left(A_{i}\right)$ tends weakly to the unbounded operator $-\log \varrho_{1 \Lambda}$. Hence for $i$ large enough

$$
\varrho_{1}\left(A_{i}\right)=\operatorname{Tr}_{\mathscr{H}_{F}(\Lambda)}\left(\varrho_{1 \Lambda} \pi_{\Lambda}\left(A_{i}\right)\right)<\operatorname{Tr}_{\mathscr{H}_{F}(\Lambda)}\left(\varrho_{2 \Lambda} \pi_{\Lambda}\left(A_{i}\right)\right)=\varrho_{2}\left(A_{i}\right),
$$

i.e. there is an $A \in \mathfrak{A}$ such that $\varrho_{1}(A) \neq \varrho_{2}(A)$, which implies the extremal invariant states $\varrho_{1}$ and $\varrho_{2}$ generate inequivalent representations.

Thus we see that by restricting our attention to the subset of states $F_{N} \cap F_{T}$ we can discuss the mean entropy in a satisfying manner. We leave open the question whether $S$ is an isomorphy invariant i.e. whether $S\left(\varrho_{1}\right) \neq S\left(\varrho_{2}\right)$ for $\varrho_{1}, \varrho_{2} \in F_{N} \cap F_{T}$ implies that $\pi_{\varrho_{1}}$ and $\pi_{\varrho_{2}}$ are unitarily inequivalent.

Acknowledgements. An earlier version of section 5 was improved by helpful remarks of H. Araki, O. Lanford and D. Ruelle, to whom we are indebted. 


\section{References}

1. Doplicher, S., D. Kastler, and D. W. Robinson:Commun. Math.Phys.3,1-28(1966).

2. Kastler, D., and D. W. Robinson: Commun. Math. Phys. 3, 151-180 (1966).

3. Ruelle, D.: Commun. Math. Phys. 3, 133-150 (1966).

4. Powers, R.: Princeton University, thesis (1967).

5. Neumann, J. von: Collected works, p. 64. Oxford: Pergamon 1961.

6. Dell'Antonio, G. F., S. Doplicher, and D. Ruelle: Commun. Math. Phys. 2, 223 to 230 (1966).

7. - - J. Math. Phys. 8, 633-666 (1967).

8. Chaiken, J. M.: Ann. Phys. 42, 23-80 (1967).

9. - Commun. Math. Phys. 8, 164-184 (1968).

10. Lanford, O. E., and D. W. Robinson: J. Math. Phys. 9, 1120-1125 (1968).

11. -, and D. Ruelle: J. Math. Phys. 8, 1460-1463 (1967).

12. Bourbaki, N.: Intégration, Chap. IV. Paris: Hermann 1965.

Salvador Miracle-Sole

Derek W. Robinson

C.N.R.S.

31 Chemin J. Aiguier

13 Marseille $\left(9^{\mathrm{e}}\right)$, France 